Portland State University

PDXScholar

1990

\title{
A theoretical and experimental study of the three- ring electrostatic electron lens
}

Thomas Anthony Sommer

Portland State University

Follow this and additional works at: https://pdxscholar.library.pdx.edu/open_access_etds

Part of the Physics Commons

Let us know how access to this document benefits you.

\section{Recommended Citation}

Sommer, Thomas Anthony, "A theoretical and experimental study of the three-ring electrostatic electron lens" (1990). Dissertations and Theses. Paper 4137.

https://doi.org/10.15760/etd.6020

This Thesis is brought to you for free and open access. It has been accepted for inclusion in Dissertations and Theses by an authorized administrator of PDXScholar. Please contact us if we can make this document more accessible: pdxscholar@pdx.edu. 
AN ABSTRACT OF THE THESIS OF Thomas Anthony Sommer for the

Master of Science in Physics presented July 9, 1990.

Title: A Theoretical and Experimental Study of the Three-Ring Electrostatic Electron Lens.

APPROVED BY THE MEMBERS OF THE THESIS COMMITTEE:

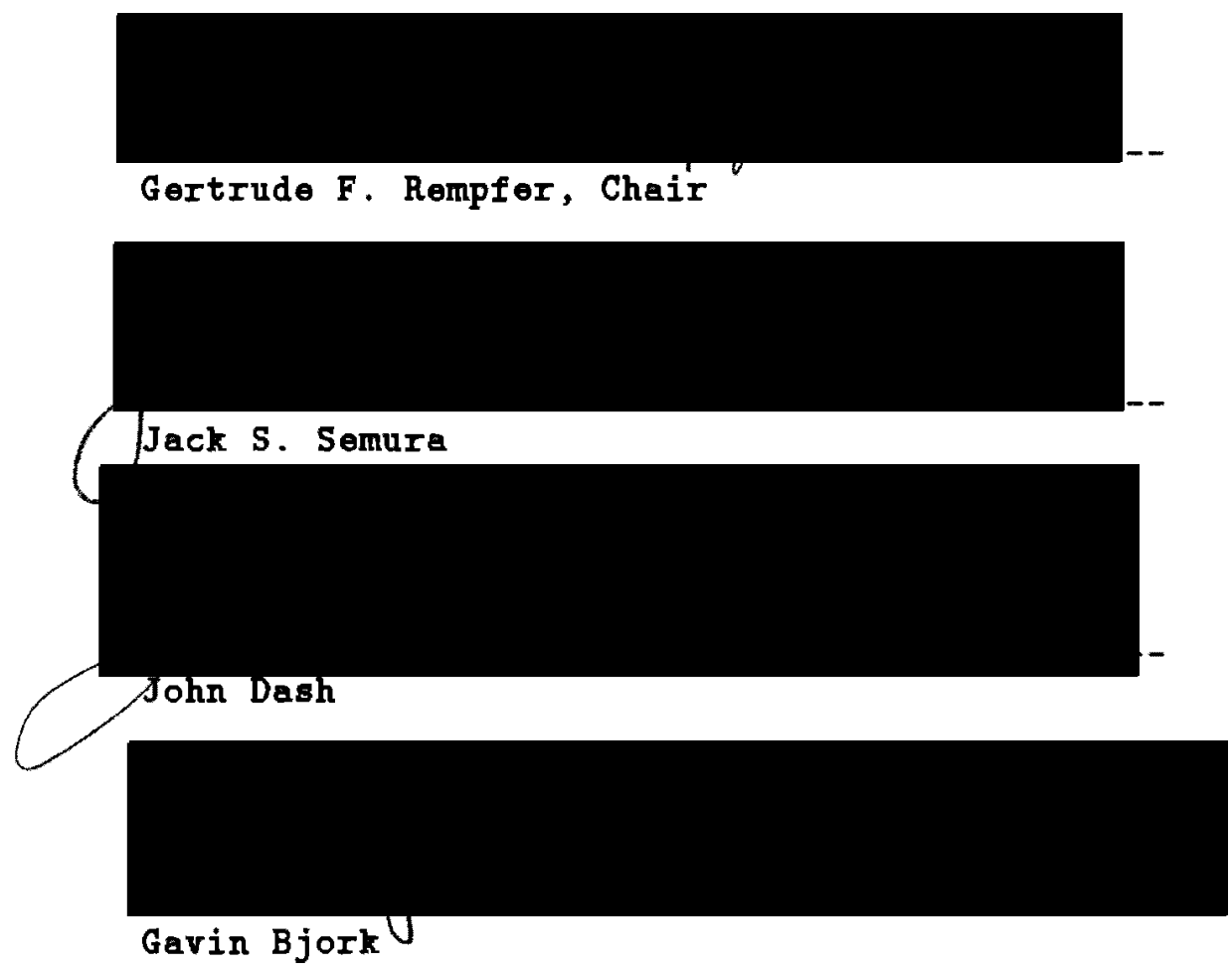

A theoretical and experimental study of the three-ring electrostatic

lens is presented. The lens consists of three isolated ring-shaped 
conductors, equally spaced along a common axis of symmetry. When appropriate potentials are applied to the conductors an electric field is produced near the axis which is capable of focusing a collimated beam of electrons. In the theoretical study the charge density method is used to find a closed-form solution for the field. This method approximates the field by replacing each ring with an infinitely thin hoop of uniform charge. The radial and axial equations of motion are then solved numerically, and the paraxial values of focal length, focal distance and their second order aberrations are found. In the experimental study these focal quantities are determined by a ray tracing method that uses tro grids placed in the bean path outside the field of the lens. One grid is placed in front of the lens while the other is placed behind it. The shador pattern cast by the grids is then analyzed to find the focal properties. This method, which is independent of the type of lens being investigated, is also used on a plano-convex glass lens. Comparison of the experimental and theoretical results shors satisfactory agreement in both cases. 


\title{
A THEORETICAL AND EXPERIMENTAL STUDY OF THE THREE-RING ELECTROSTATIC ELECTRON LENS
}

\author{
by \\ THOMAS ANTHONY SOMMER
}

A thesis submitted in partial fulfillment of the requirements for the degree of

\author{
MASTER OF SCIENCE \\ in \\ PHYSICS
}

Portland State University

1990 
TO THE OPFICE OF GRADUATE STUDIES:

The members of the Committee approve the thesis of Thonas Anthony Sommer presented July 9, 1990.

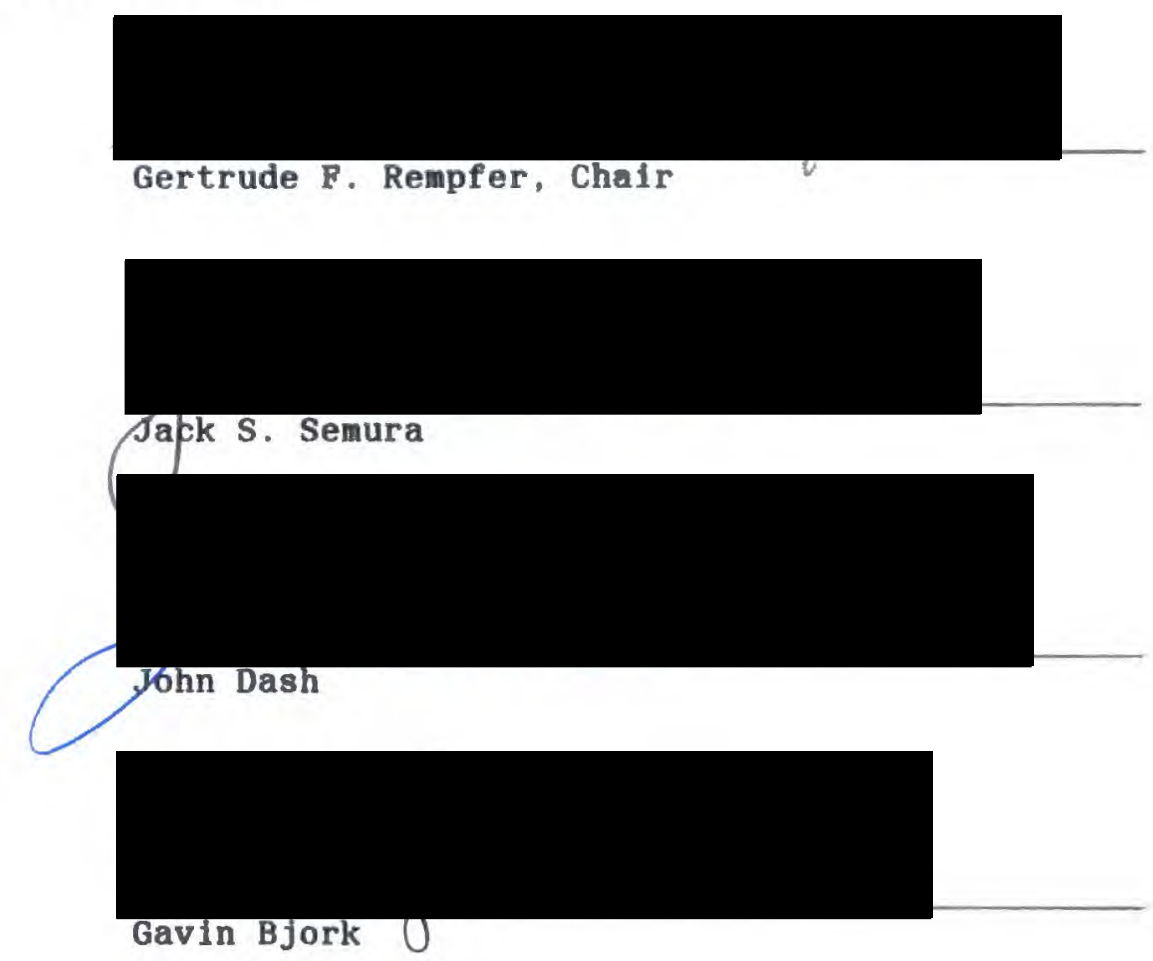

APPROVED :

Mark Gurevitch, Chair, Department of Physics

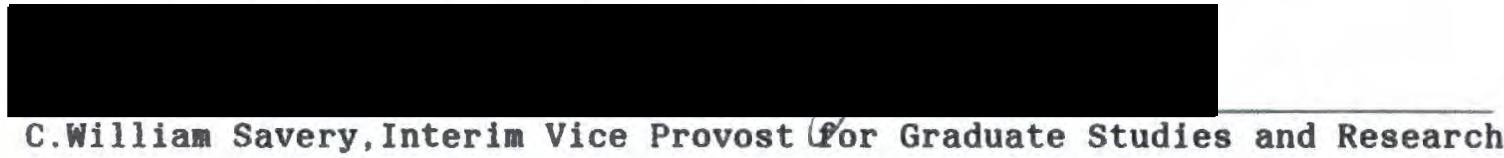




\section{TABLE DF CONTENTS}

\section{PAGE}

LIST OF TABLES . . . . . . . . . . . . . . . . . . . . . . . . . iv

LIST OF FIGURES. . . . . . . . . . . . . . . . . . . . . . . . v v

INTRODUCTION . . . . . . . . . . . . . . . . . . . . . 1

THEORETICAL STUDY. . . . . . . . . . . . . . . . . . 3

Electric Field Calculation. . . . . . . . . . . . . 3

Trajectory Calculations... . . . . . . . . . . . . 11

EXPERIMENTAL STUDY . . . . . . . . . . . . . . . . . . . . 16

Apparatus and Procedure... . . . . . . . . . . . 20

Sample Calculation and Rosults. . . . . . . . . . . . 23

CONCLUSIONS. . . . . . . . . . . . . . . . . . . . . . . . . . 33

GLASS LENS STUDY . . . . . . . . . . . . . . . . . . . . . . . . . 39

Experiment. . . . . . . . . . . . . . . . . 39

Results . . . . . . . . . . . . . . . . . . . . 41

REFERENCES . . . . . . . . . . . . . . . . . . . . . . . . 43 


\section{LIST OF FIGURES}

FIGURE

PAGE

1. Three-ring Lens . . . . . . . . . . . . . . . . . . . . . . . 4

2. Lens Parameters. . . . . . . . . . . . . . . . . . . . . . 5

3. Potential due to a Hoop of Charge . . . . . . . . . . . . . . 7

4. Path through a Slice. . . . . . . . . . . . . . . . . . . . . 13

5. Optical System Ray Trace Parameters . . . . . . . . . . . . . 17

6. Ray Trace for a Distant Source. . . . . . . . . . . . . . . . 19

7. Electron Optical Bench with Components. . . . . . . . . . . . 21

8. Three-ring Lens Disassembled. . . . . . . . . . . . . . . . . 22

9. Scale Draring of Lens Assembly. . . . . . . . . . . . . . . . 24

10. Grating Shador Pattern. . . . . . . . . . . . . . . . . . . 25

11. Method of Converting $n$ ' to n. . . . . . . . . . . . . . . 28

12. Magnification Plot for Front Grating. . . . . . . . . . . . . 29

13. Magnification Plot for Rear Grating . . . . . . . . . . . . . 30

14. Theoretical and Experimental Focal Length . . . . . . . . . . 34

15. Theoretical and Experimental Focal Distance. . . . . . . . . 35

16. Theoretical and Experimental Focal Length Aberration. . . . . 36

17. Theoretical and Experimental Focal Distance Aberration. . . . 37

18. Experimental Apparatus for Glass Lens Study . . . . . . . . . 40

19. Experimental Components for Glass Lens Study. . . . . . . . . 40 


\section{LIST OF TABLES}

TABLE

PAGE

I Theoretical focal parameters . . . . . . . . . . . 15

II Experimental data. . . . . . . . . . . . . . . . 26

III Experimental focal parameters. . . . . . . . . . . . . 32

IV Glass Lens Results . . . . . . . . . . . . . . . . . 42 


\section{INTRODUCTION}

Much of the science of electron optics depends on the fact that particles moving in a field of force can be focused in the same way that rays of light are focused by a glass lens. This idea was first examined in 1828 when Hamilton [1] brought attention to similarities between the equations that describe light rays passing through a medium whose index of refraction varies in space and those that describe a particle moving in a force field. The analogy was demonstrated experimentally in 1926 by H. Busch [2] who used an axially symmetric magnetic field generated by a short coil to focus electrons, and this was followed in the early 1930's by similar experiments that used electric fields to accomplish the same results. The dual nature of waves and particles was addressed theoretically in a paper by De Broglie [3] in 1924, and in 1925 Davisson and Germer [4] observed diffraction effects from an electron beam incident on a nickel crystal, verifying De Broglie's hypothesis. These breakthroughs provided the experimental and theoretical groundwork for the development of the many electron optical devices in use today. Some of these devices, such as the electron microscopes, require high imaging reliability which in turn depends on the focal properties of the electron lenses used in them. Therefore, there is continued interest in the study of electron lenses and the aberrations exhibited by them which requires a detailed understanding of their electric and magnetic fields. For electrostatic lenses, such as the one considered in this paper, this involves solving Laplace's equation for a given set of electrode surfaces and potentials. The method used in this paper to accomplish this is the charge density method described by Renau, Read and Brunt [5]. It has been used for the study of electron optical systems since $1963[6]$ and has been further developed for this purpose up through the 
early 1980's [7]. The method has several advantages over the older relaxation techniques $[8]$ which provide potentials only at discrete points in space, have a slower convergence and require a large number of mesh points to give accurate solutions. When the charge density method is applied to systems with cylindrical symmetry the electrode surfaces are usually broken up into many thin flat rings of uniform charge. A set of coupled linear equations is then generated by requiring appropriate potentials on each ring in the presence of the other rings and the equations are solved to find the charge on each ring. Finally, the rings are used with their charge values to find the electric field. Normally this procedure requires the use of a digital computer to solve the set of equations that determines the charge values but the three-ring lens possesses a particularly simple electrode geometry which allows an approximate closed-form expression to be found for the field near the axis. This expression is derived and used in trajectory calculations which are used to find the focal properties of the lens. The lens is then built and tested experimentally to verify the predicted values. This study, therefore, provides a simple and illustrative example of an electric field calculation using the charge density method. 


\section{THEORETICAL STUDY}

In this study the theoretical focal properties of the three-ring electron lens are determined. This is done by finding the electric field formed by the lens near its axis (taking advantage of the planar and axial symmetry of the problem) and then using Newton's second law of motion to calculate the trajectories of the electrons on a digital computer. The parameters of the problem are shown in figures (1) and (2). The field is found by using a variation of the charge density method, a method in which the electrodes are replaced by thin strips of charge and the electric potential is found from the values of the charges. The potential is expanded as a function of the radial distance and a near-axis approximation for the field is found. Finally, the trajectories are calculated by dividing the space of the lens into a large number of cylindrical "slices", each having a constant field, and the equations of motion are worked out in general for a slice, in a form suitable for use on a computer. The focal properties are calculated for several ratios of cathode voltage to lens voltage, $V_{c} / V_{l}$. The assumptions used are: 1) the electrons are confined to a region near the axis of the lens and 2) polarization of charge on the electrodes of the lens has a small effect on the trajectories.

\section{ELECTRIC FIELD CALCULATION}

The approach for finding the field is to replace each ring-shaped conductor with an infinitely thin hoop of uniform charge, located at the center of the ring. The values of the charges are then found by requiring the appropriate potentials at selected locations which correspond to the portions of the surfaces of the rings which are closest to the axis of symmetry. The first step is to find the potential in 


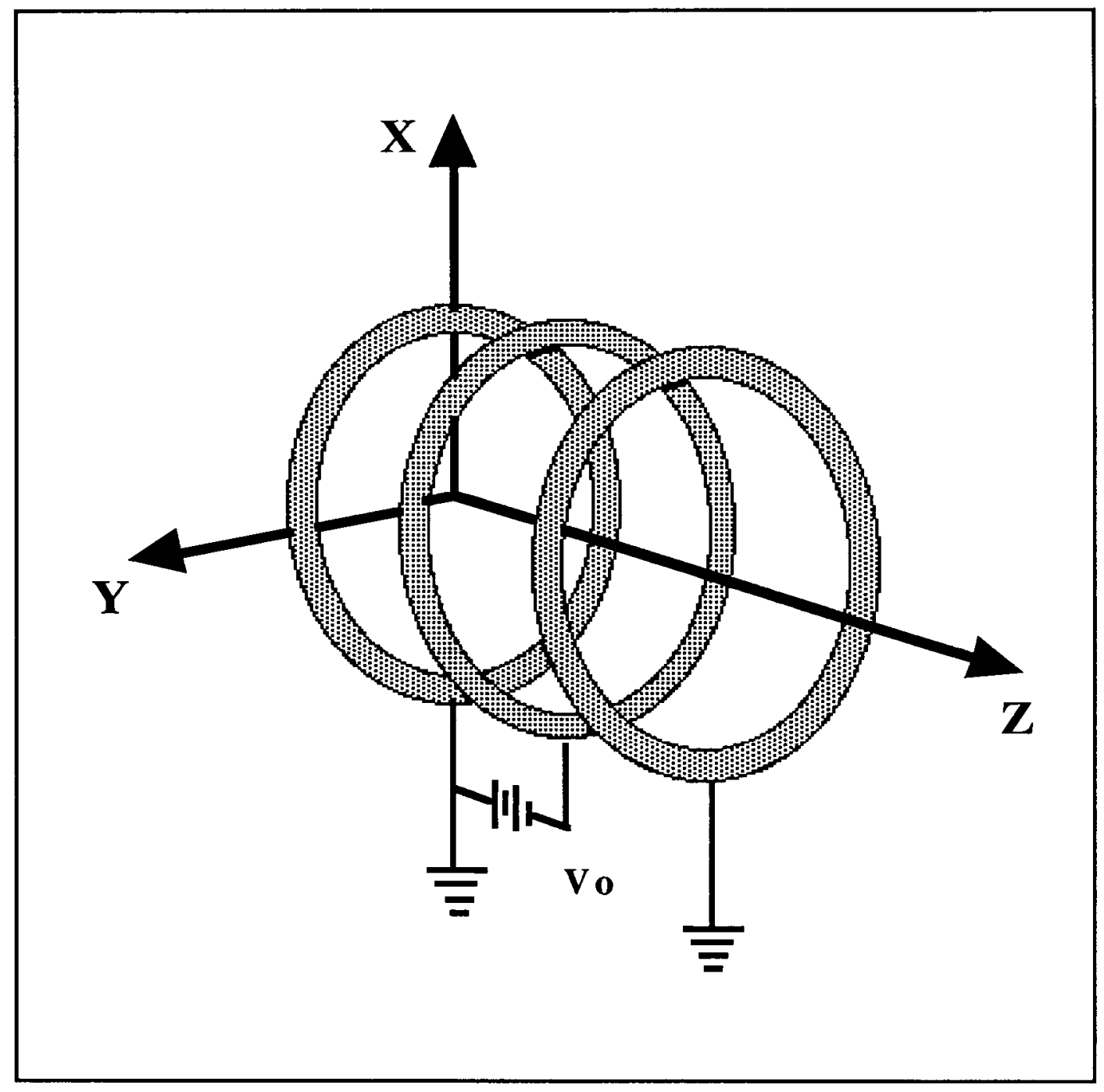

Figure 1. The three-ring lens: 3D view. 


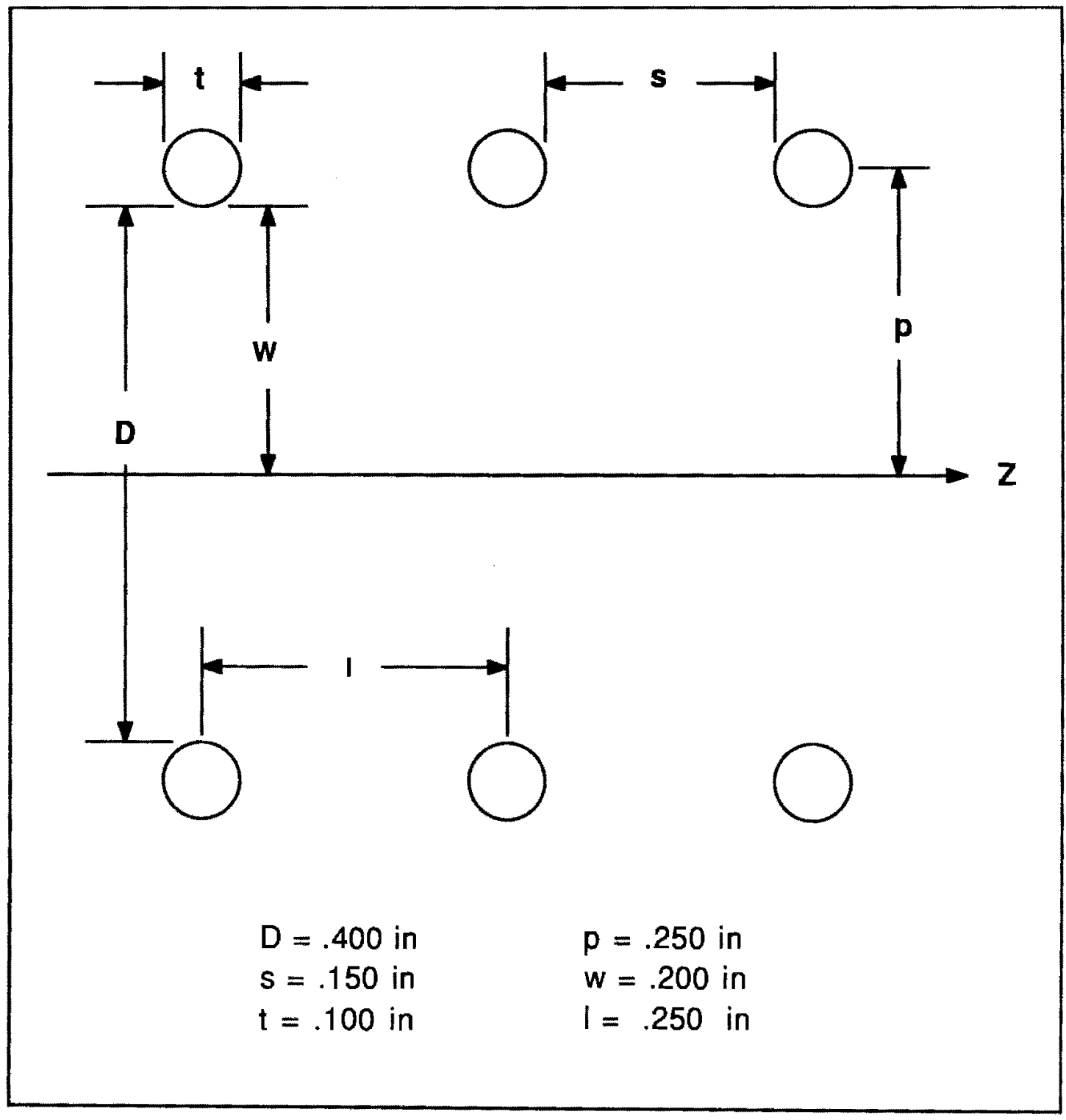

Eigure 2. Three-ring lens parameters: cross section view. 
space due to a hoop of charge $q$ whose radius is $p$ (which is the mean radius of a ring as shown in figure (2)) and whose center is located at the origin. The problem has cylindrical symmetry so cylindrical coordinates are used. The potential is described by

$$
V(\rho, \phi, z)=\int \frac{d q}{\left|\mathbf{r}-\mathbf{r}^{\prime}\right|}
$$

where $\mathbf{r}^{\prime}$ is the vector that points to the element of charge $d q$ and $\mathbf{r}$ is the vector that points to the location where the potential is being determined. In this case $d q=\lambda d \phi^{\prime}$ where $\phi^{\prime}$ is the angle of integration and $\lambda=q / 2 \pi$. These variables are depicted in figure (3) with a perspective view. For the cylindrical symmetry shown

$$
\mathbf{r}^{\prime}=p \cos \phi^{\prime} \hat{\imath}+p \sin \phi^{\prime} \hat{\jmath}
$$

the hoop is at $z=0$ but the point $P$ is a distance $z$ from the $x-y$ plane so that

$$
\mathbf{r}=\rho \cos \phi \hat{\imath}+\rho \sin \phi \hat{\jmath}+z \hat{k}
$$

and

$$
\left|\mathbf{r}-\mathbf{r}^{\prime}\right|=\sqrt{p^{2}+\rho^{2}+z^{2}-2 p \rho \cos \left(\phi-\phi^{\prime}\right)}
$$

This yields

$$
V(\rho, \phi, z)=\frac{1}{4 \pi \epsilon_{0}} \frac{q}{2 \pi} \int_{0}^{2 \pi} \frac{d \phi^{\prime}}{\sqrt{p^{2}+\rho^{2}+z^{2}-2 p \rho \cos \left(\phi-\phi^{\prime}\right)}}
$$

setting $\phi=0$, substituting $\theta=\phi^{\prime} / 2$ and using the trig identity $\cos 2 \theta=2 \cos ^{2} \theta-1$ gives

$$
\frac{1}{4 \pi \epsilon_{0}} \frac{2 q}{\pi} \int_{0}^{\pi / 2} \frac{d \theta}{\sqrt{(p+\rho)^{2}+z^{2}-4 p \rho \cos ^{2} \theta}}
$$

and now substituting $\psi=\pi / 2-\theta$ and using the identity $\cos (\pi / 2-\theta)=\sin \theta$ gives

$$
\frac{1}{4 \pi \epsilon_{0}} \frac{2 q}{\pi} \int_{0}^{\pi / 2} \frac{d \psi}{\sqrt{(p+\rho)^{2}+z^{2}-4 p \rho \sin ^{2} \psi}}
$$




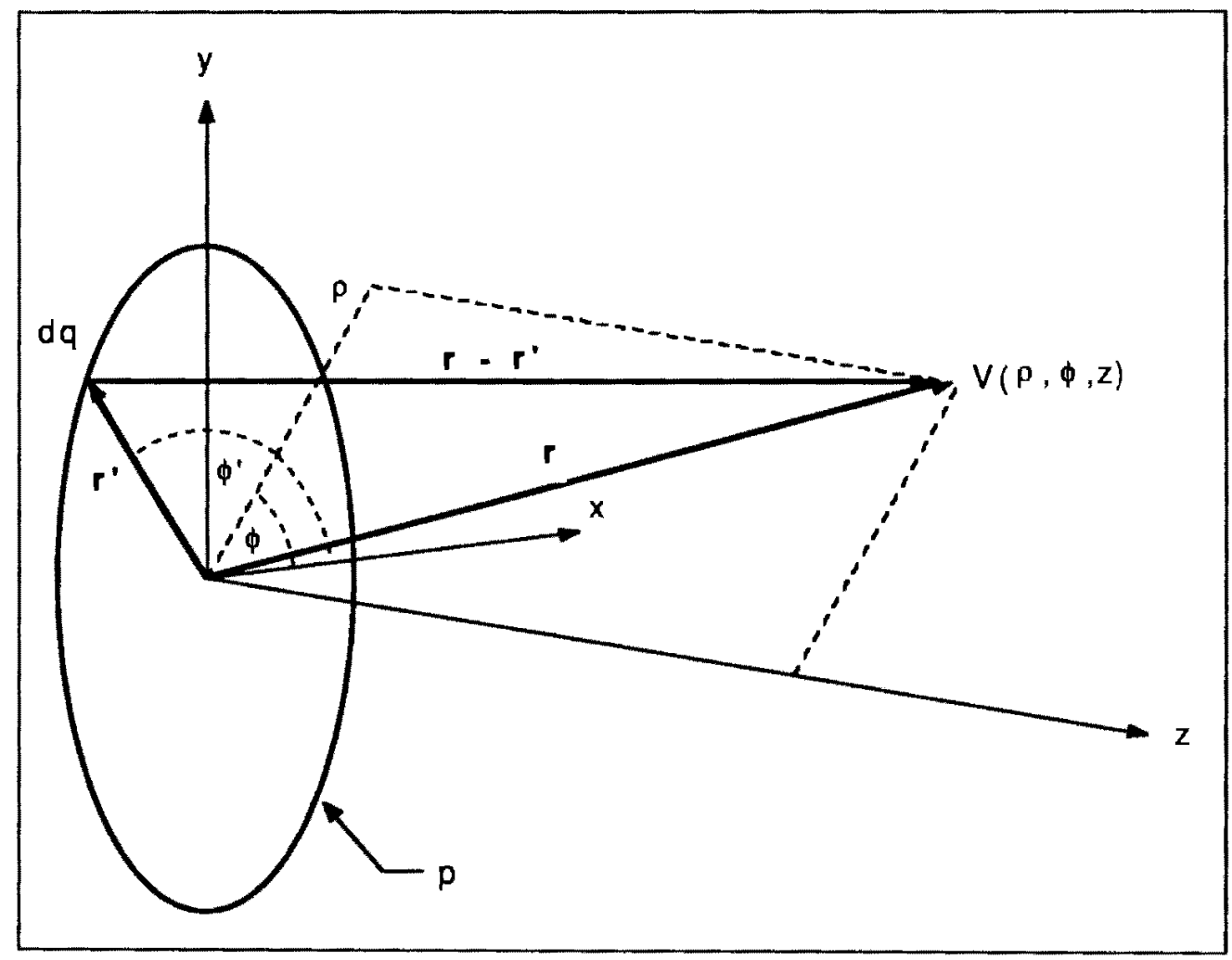

Figure 3. Potential due to a hoop of uniform charge 
which becomes

$$
\frac{1}{4 \pi \epsilon_{0} \pi \sqrt{p \rho}} k \int_{0}^{\frac{\pi}{2}} \frac{d \psi}{\sqrt{1-k^{2} \sin ^{2} \psi}}
$$

where

$$
k=\left[\frac{4 \rho / p}{(1+\rho / p)^{2}+(z / p)^{2}}\right]^{\frac{1}{2}}
$$

and the integral is a complete elliptic integral of the first kind, written from here on as $K(k)$ so that

$$
V(\rho, z)=\frac{q}{4 \pi^{2} \epsilon_{0} \sqrt{p \rho}} k K(k)
$$

Now the values of the charges needed to simulate the rings can be found. A point on each conductor surface, which is closest to the axis, is chosen and the potential at each of these points is required to be equal to the voltage, $V_{i}$, on the conductor that corresponds to it. The general equations for a system of conductors are

$$
\begin{aligned}
& V_{i}=\sum_{j} V_{i j} \\
& V_{i j}=q_{j} A_{i j}
\end{aligned}
$$

where $V_{i j}$ is the potential on the $i^{\text {th }}$ conductor due to the $j^{\text {th }}$ conductor and $A_{i j}$ is a geometrical constant. For the three hoops this becomes

$$
\begin{gathered}
V_{1}=0=V_{11}+V_{12}+V_{13}=q_{1} A_{11}+q_{2} A_{12}+q_{3} A_{13} \\
V_{2}=-V_{0}=V_{21}+V_{22}+V_{23}=q_{1} A_{21}+q_{2} A_{22}+q_{3} A_{23} \\
V_{3}=0=V_{31}+V_{32}+V_{33}=q_{1} A_{31}+q_{2} A_{32}+q_{3} A_{33}
\end{gathered}
$$

Since the system is symmetric about the midplane and the conductors are identical

$$
\begin{gathered}
q_{1}=q_{3} \\
A_{11}=A_{22}=A_{33} \\
A_{12}=A_{21}=A_{23}=A_{32}
\end{gathered}
$$




$$
A_{13}=A_{31}
$$

and equations (13), (14) and (15) can be rewritten as

$$
\begin{gathered}
q_{1} A_{11}+q_{2} A_{12}+q_{1} A_{13}=0 \\
q_{1} A_{12}+q_{2} A_{11}+q_{1} A_{12}=-V_{0} \\
q_{1} A_{13}+q_{2} A_{12}+q_{1} A_{11}=0
\end{gathered}
$$

where equations (20) and (22) are equivalent. Solving the first two gives

$$
\begin{gathered}
q_{1}=\frac{A_{12}}{A_{11}\left(A_{11}+A_{13}\right)-2 A_{12}^{2}} V_{0} \\
q_{2}=-\frac{A_{11}+A_{13}}{A_{11}\left(A_{11}+A_{13}\right)-2 A_{12}^{2}} V_{0}
\end{gathered}
$$

where

$$
A_{i j}=\frac{1}{4 \pi \epsilon_{0}} \frac{1}{2 \pi} \int_{0}^{2 \pi} \frac{d \phi^{\prime}}{\sqrt{p^{2}+w^{2}+\left(z_{i}-z_{j}\right)^{2}-2 p w \cos \left(\phi-\phi^{\prime}\right)}}
$$

where $w$ is the inner radius of the electrodes (which is where the potentials due to the charges are required to agree with the voltages on the lens), $p$ is the radius of the hoops of charge and the subscripts refer to the positions of the two conductors along the $z$ axis. Using the result previously worked out for a hoop of charge this becomes

$$
A_{i j}=\frac{1}{4 \pi^{2} \epsilon_{0} \sqrt{p w}} k_{i j} K\left(k_{i j}\right)
$$

where

$$
k_{i j}=\sqrt{\frac{4(w / p)}{(1+(w / p))^{2}+\left(\left(z_{i}-z_{j}\right) / p\right)^{2}}}
$$

which for $w / p=.80$ and $\left(z_{2}-z_{1}\right) / p=l / p=1.0$ gives

$$
A_{11}=\frac{1}{4 \pi \epsilon_{0} \sqrt{p w}}(1.1348)
$$




$$
\begin{aligned}
& A_{12}=\frac{1}{4 \pi \epsilon_{0} \sqrt{p w}}(.59871) \\
& A_{13}=\frac{1}{4 \pi \epsilon_{0} \sqrt{p w}}(.38258)
\end{aligned}
$$

and finally since $w=.200$ inches and $p=.250$ inches

$$
\begin{gathered}
q_{1}=q_{3}=4 \pi \epsilon_{0} p(.5329) V_{0}=-3.763 \times 10^{-13} \frac{\text { coulombs }}{\text { volt }}\left(V_{0}\right) \\
q_{2}=-4 \pi \epsilon_{0} p(1.351) V_{0}=9.541 \times 10^{-13} \frac{\text { coulombs }}{\text { volt }}\left(V_{0}\right)
\end{gathered}
$$

and now the potential in space due to all three hoops is given by

$$
V(\rho, z)=q_{1} B_{1}+q_{2} B_{2}+q_{3} B_{3}
$$

where

$$
B_{i}=\frac{1}{4 \pi^{2} \epsilon_{0} \sqrt{p \rho}} k_{i} K\left(k_{i}\right)
$$

and

$$
k_{i}=\sqrt{\frac{4 \rho / p}{(1+\rho / p)^{2}+\left(\left(z-z_{i}\right) / p\right)^{2}}}
$$

This can be used with the identity $\mathbf{E}=-\nabla V$ to give the electric field at all points in space (outside the conductors) in terms of complete elliptic integrals of the first and second kind, but it is only necessary to know the field near the axis which is found by using the series expansion

$$
V(\rho, z)=V(0, z)-\frac{\rho^{2}}{4} \frac{\partial^{2} V(0, z)}{\partial z^{2}}+\frac{\rho^{4}}{64} \frac{\partial^{4} V(0, z)}{\partial z^{4}}-\cdots
$$

together with the on-axis potential due to one hoop of charge at the origin

$$
V(0, z)=\frac{q}{4 \pi \epsilon_{0} \sqrt{p^{2}+z^{2}}}
$$

keeping the first three terms of the equation (35) gives the approximation

$$
\begin{aligned}
V(\rho, z) & \approx \frac{q}{4 \pi \epsilon_{0} \sqrt{p^{2}+z^{2}}}\left[1+\frac{\rho^{2}}{4}\left(\frac{1}{\left(p^{2}+z^{2}\right)}-\frac{3 z^{2}}{\left(p^{2}+z^{2}\right)^{2}}\right)\right. \\
& \left.+\frac{\rho^{4}}{64}\left(\frac{9}{\left(p^{2}+z^{2}\right)^{2}}-\frac{90 z^{2}}{\left(p^{2}+z^{2}\right)^{3}}+\frac{105 z^{4}}{\left(p^{2}+z^{2}\right)^{4}}\right)\right]
\end{aligned}
$$


and the approximate field for one ring at the origin is

$$
\begin{aligned}
E_{\rho}=-\frac{\partial V}{\partial \rho} & \approx-\frac{q}{4 \pi \epsilon_{0} \sqrt{p^{2}+z^{2}}}\left[\frac{\rho}{2}\left(\frac{1}{\left(p^{2}+z^{2}\right)}-\frac{3 z^{2}}{\left(p^{2}+z^{2}\right)^{2}}\right)\right. \\
& \left.+\frac{\rho^{3}}{16}\left(\frac{9}{\left(p^{2}+z^{2}\right)^{2}}-\frac{90 z^{2}}{\left(p^{2}+z^{2}\right)^{3}}+\frac{105 z^{4}}{\left(p^{2}+z^{2}\right)^{4}}\right)\right] \\
E_{z}= & -\frac{\partial V}{\partial z} \approx-\frac{\partial V(0, z)}{\partial z}=\frac{q}{4 \pi \epsilon_{0}} \frac{z}{\left(p^{2}+z^{2}\right)^{3 / 2}}
\end{aligned}
$$

now if the origin is chosen to be at the center of the lens then $z_{1}=-l, z_{2}=0$,and $z_{3}=l$ and the total paraxial field for the three-ring lens is

$$
\begin{aligned}
E_{\rho} & \approx-\frac{1}{4 \pi \epsilon_{0}} \sum_{i=1}^{3} \frac{q_{i}}{\sqrt{p^{2}+\left(z-z_{i}\right)^{2}}}\left[\frac{\rho}{2}\left(\frac{1}{\left(p^{2}+\left(z-z_{i}\right)^{2}\right)}-\frac{3\left(z-z_{i}\right)^{2}}{\left(p^{2}+\left(z-z_{i}\right)^{2}\right)^{2}}\right)\right. \\
& \left.+\frac{\rho^{3}}{16}\left(\frac{9}{\left(p^{2}+\left(z-z_{i}\right)^{2}\right)^{2}}-\frac{90\left(z-z_{i}\right)^{2}}{\left(p^{2}+\left(z-z_{i}\right)^{2}\right)^{3}}+\frac{105\left(z-z_{i}\right)^{4}}{\left(p^{2}+\left(z-z_{i}\right)^{2}\right)^{4}}\right)\right] \\
E_{z} & \approx \frac{1}{4 \pi \epsilon_{0}} \sum_{i=1}^{3} q_{i} \frac{z-z_{i}}{\left(p^{2}+\left(z-z_{i}\right)^{2}\right)^{3 / 2}}
\end{aligned}
$$

where $q_{1}, q_{2}$ and $q_{3}$ come from equations (31) and (32).

\section{TRAJECTORY CALCULATIONS}

Once the electric field is known the next step is to find the electron trajectories. It is assumed that the electrons are originally traveling parallel to the axis and are located a small distance $\rho<<a$ from the axis. The initial velocity is determined from the energy equation $-e V_{c}=\frac{1}{2} m v^{2}$ which becomes

$$
\begin{gathered}
v_{\rho}=0 \\
v_{z}=\sqrt{-\frac{2 e V_{\mathrm{c}}}{m}}
\end{gathered}
$$

where $V_{c}$ is the cathode voltage, $m$ is the electron mass and $e$ is the magnitude of the electron charge. Now the field is approximated by cutting the space of the 
lens into thin cylindrical "slices" and then treating each slice as having a constant field, $E_{\rho}$ and $E_{z}$, which is evaluated at the point where the electron would cross the center of the slice if no forces acted on it (see figure 4). From Newton's second law of motion, $\mathbf{F}=m \mathbf{a}$, and the assumption that there is no $\hat{\phi}$ component in the velocity

$$
\begin{aligned}
& F_{p}=m \ddot{a}=-e E_{p} \\
& F_{z}=m \ddot{z}=-e E_{z}
\end{aligned}
$$

and this immediately leads to the set of equations that describe the motion in a given slice (the $i^{\text {th }}$ slice) of the field.

$$
\begin{gathered}
\rho^{i+1}=\rho^{i}+v_{\rho}^{i} t^{i}+\frac{1}{2} A_{\rho}^{i}\left(t^{i}\right)^{2} \\
z^{i+1}=z^{i}+v_{z}^{i} t^{i}+\frac{1}{2} A_{z}^{i}\left(t^{i}\right)^{2} \\
v_{\rho}^{i+1}=v_{\rho}^{i}+A_{\rho}^{i} t^{i} \\
v_{z}^{i+1}=v_{z}^{i}+A_{z}^{i} t^{i}
\end{gathered}
$$

where

$$
\begin{aligned}
A_{\rho}^{i} & =-\frac{e}{m} E_{\rho}^{i} \\
A_{\mathrm{z}}^{i} & =-\frac{e}{m} E_{\mathrm{z}}^{i}
\end{aligned}
$$

since the width of the slice,$z^{i+1}-z^{i}$, is known, $t^{i}$ can be found from equation (47)

$$
t^{i}= \begin{cases}-\frac{v_{z}^{i}}{A_{z}^{i}}+\sqrt{\left(\frac{v_{x}^{i}}{A_{z}^{i}}\right)^{2}+2 \frac{\left(z^{i+1}-z^{i}\right)}{A_{z}^{i}}} & \text { if } A_{z}^{i}>0 ; \\ -\frac{v_{i}^{i}}{A_{z}^{i}}-\sqrt{\left(\frac{v_{t}^{i}}{A_{z}^{i}}\right)^{2}+2 \frac{\left(z^{i+1}-z^{i}\right)}{A_{z}^{i}}} & \text { otherwise. }\end{cases}
$$

where the postive root for $A_{z}^{i}<0$ is not included because it corresponds to the case where the electron is reflected, which does not occur in this problem. Now $t^{i}$ can 


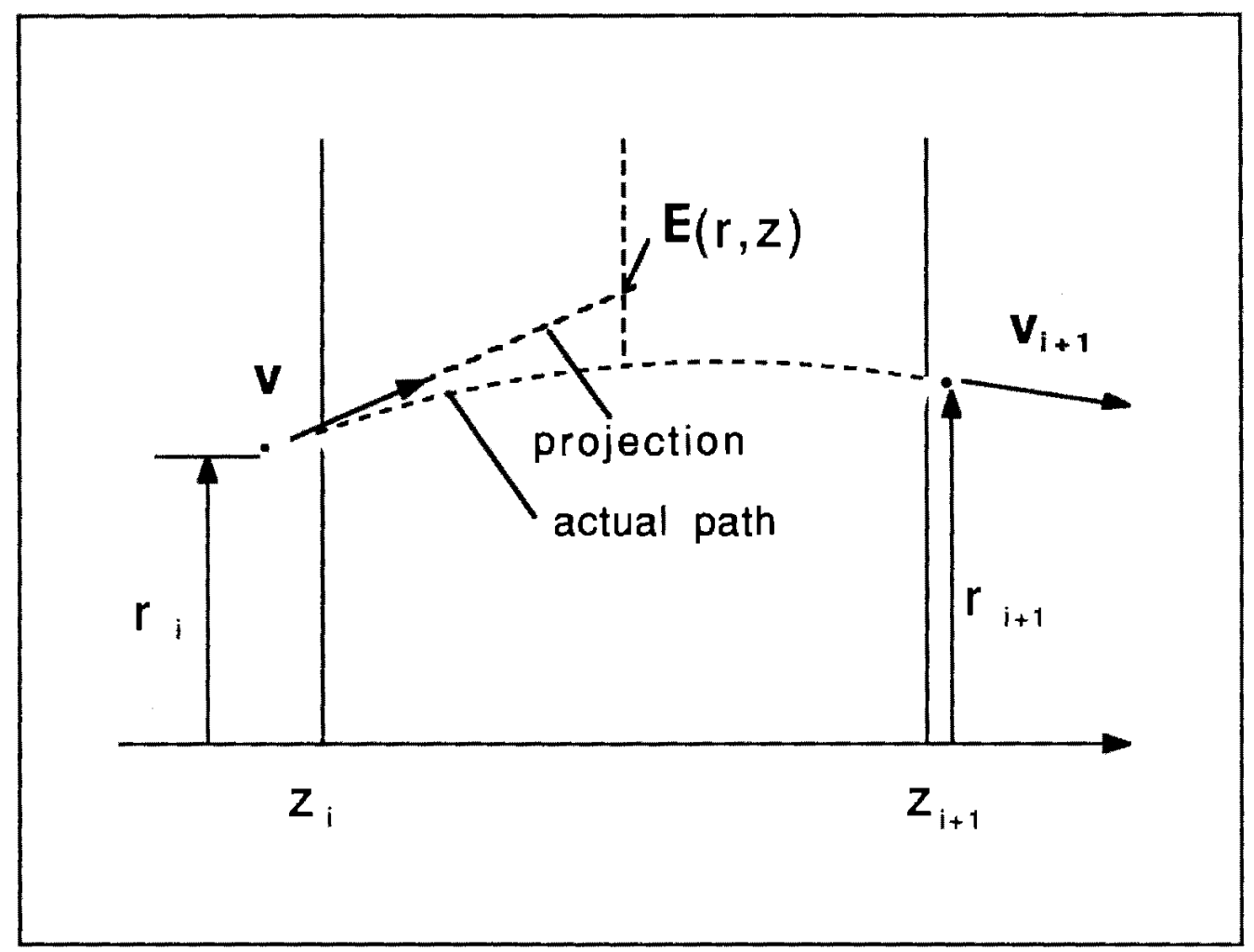

Figure 4. Path through a slice of the lens field. 
be used in equations $(47),(49)$ and (50) to update $\rho, v_{\rho}$ and $v_{z}$ for each slice iteratively throughout the field of the lens to give the trajectory as a function of time.

For the results presented in table (I) the lens field is divided into 10,000 sections and the equations of motion are solved for each section. Once the electron is outside the field of the lens, its final direction is projected back onto the axis to find the focal point. This is repeated for ten rays entering at different radial distances. Thus the focal length and focal distance are found as a function of the radial height of the ray at the principle surface, $\rho$. The paraxial values, $f_{0}$ and $g_{0}$, and their second order aberrations, $S_{f}$ and $S_{g}$, are then found from these functions as defined by the equations

$$
\begin{aligned}
& \frac{\Delta f}{f_{0}}=\frac{S_{f} \rho^{2}}{f_{0}^{2}} \\
& \frac{\Delta g}{f_{0}}=\frac{S_{g} \rho^{2}}{f_{0}^{2}}
\end{aligned}
$$


TABLE I

THEORETICAL FOCAL PARAMETERS

\begin{tabular}{|c||c|c|c|c|}
\hline$V_{l} / V_{c}$ & $f_{0}($ in) & $g_{0}$ (in) & $S_{f}$ & $S_{g}$ \\
\hline \hline 1.00 & 1.25 & 1.22 & -77.6 & -78.4 \\
\hline 0.95 & 1.46 & 1.44 & -103 & -104 \\
\hline 0.90 & 1.72 & 1.70 & -137 & -138 \\
\hline 0.85 & 2.04 & 2.02 & -185 & -186 \\
\hline 0.80 & 2.42 & 2.40 & -253 & -254 \\
\hline 0.75 & 2.90 & 2.88 & -350 & -351 \\
\hline
\end{tabular}




\section{EXPERIMENTAL STUDY}

In this study the two-grating method of Spangenburg and Field [9] is used to determine the focal properties of the electron lens. This method, which is extended by Rempfer [10] to include calculation of the spherical aberrations, consists of placing two fine wire gratings in the beam path outside the lens and analyzing the magnified shadow pattern cast by them on photographic film. One grating is placed in the field free space in front of the lens and the other in the field free space behind the lens. The gratings are rotated relative to each other by ninety degrees so that the patterns formed by them are distinguishable from each other. Thus the rays in the object and image space of the lens can be traced and the focal properties determined. The experimental arrangement and parameters are shown in figure (5) which shows a ray coming from a small source a distance $z$ away from the center of the lens, passing through the lens, forming a demagnified image at $z^{\prime}$ and finally falling on a viewing screen some distance away. The front grating is a distance $a$ from the source, and the back grating is a distance $b$ from the center of the lens and $d$ from the viewing screen. The lens is a unipotential lens with symmetery about its midplane, therefore the center is also the location of the reference plane which is chosen to be midway between its focal planes. The height of a given ray from the axis as it passes through a grating is $e$ for the front grating and $e^{\prime}$ for the back grating, and $E$ is its height as it reaches the viewing screen. The image magnification therefore is

$$
m=\frac{\alpha}{\alpha^{\prime}}=\left(\frac{M^{\prime}}{M}\right)\left(\frac{c}{a}\right)
$$

where 


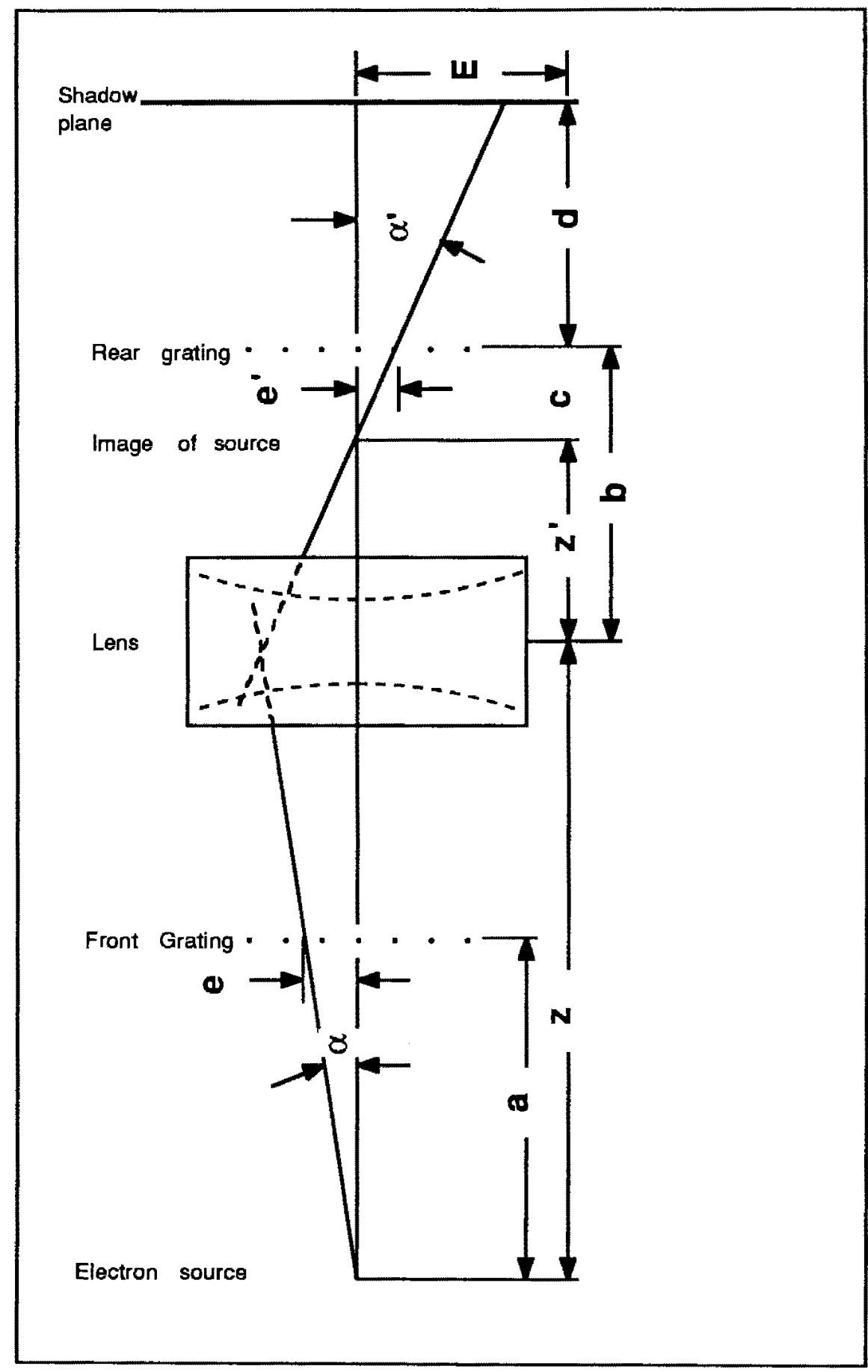

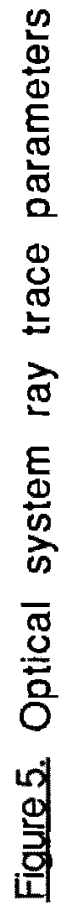




$$
c=b-z^{\prime}=\frac{d}{M^{\prime}-1}
$$

where $M=E / e$ and $M^{\prime}=E / e^{\prime}$. In this study it is assumed that the spacings in each grating are equal, i.e. $e=n e_{1}$ where $e_{1}$ is the spacing between two consecutive wires in a grating. The case where the source is distant and the incoming rays are parallel to the axis is shown in figure (6), which defines the nomenclature for the focal properties of the lens. For the general case the focal length $f$ and focal distance $g$ can be found from

$$
\begin{gathered}
f=\frac{z-z^{\prime}}{1 / m-m} \\
g=z^{\prime}-f m
\end{gathered}
$$

These quantities as a rule will vary with the height of the incoming rays due to spherical aberration, which manifests itself as distortions in the grating shadow patterns. Therefore rays entering the lens parallel to the axis at different heights will cross the axis at different positions and the principle surfaces $H$ and $H^{\prime}$ will be curved rather than planar. The spherical aberration in this experiment is predominantly second order, therefore the shadow magnifications can be expressed with sufficient accuracy by

$$
\begin{gathered}
M=M_{0}\left(1+\beta n^{2}\right) \\
\left(M^{\prime}-1\right)^{-1}=\left(M_{0}-1\right)^{-1}\left(1+\beta^{\prime} n^{2}\right)
\end{gathered}
$$

where $n$ can be found for equation (61) by plotting $E$ vs. $n$ and $n^{\prime}$, and converting $n^{\prime}$ into $n$ for a given $E$ value.

The distortion coefficients, $\beta$ and $\beta^{\prime}$, and the paraxial magnifications, $M_{0}$ and $M_{0}^{\prime}$, are found by plotting $M$ vs $n^{2}$ and $\left(M^{\prime}-1\right)^{-1}$ vs $n^{2}$. Substituting the values $M_{0}$ and $M_{0}^{\prime}$ into the previous equations gives the paraxial values $z_{0}^{\prime}, m_{0}, f_{0}$ and 


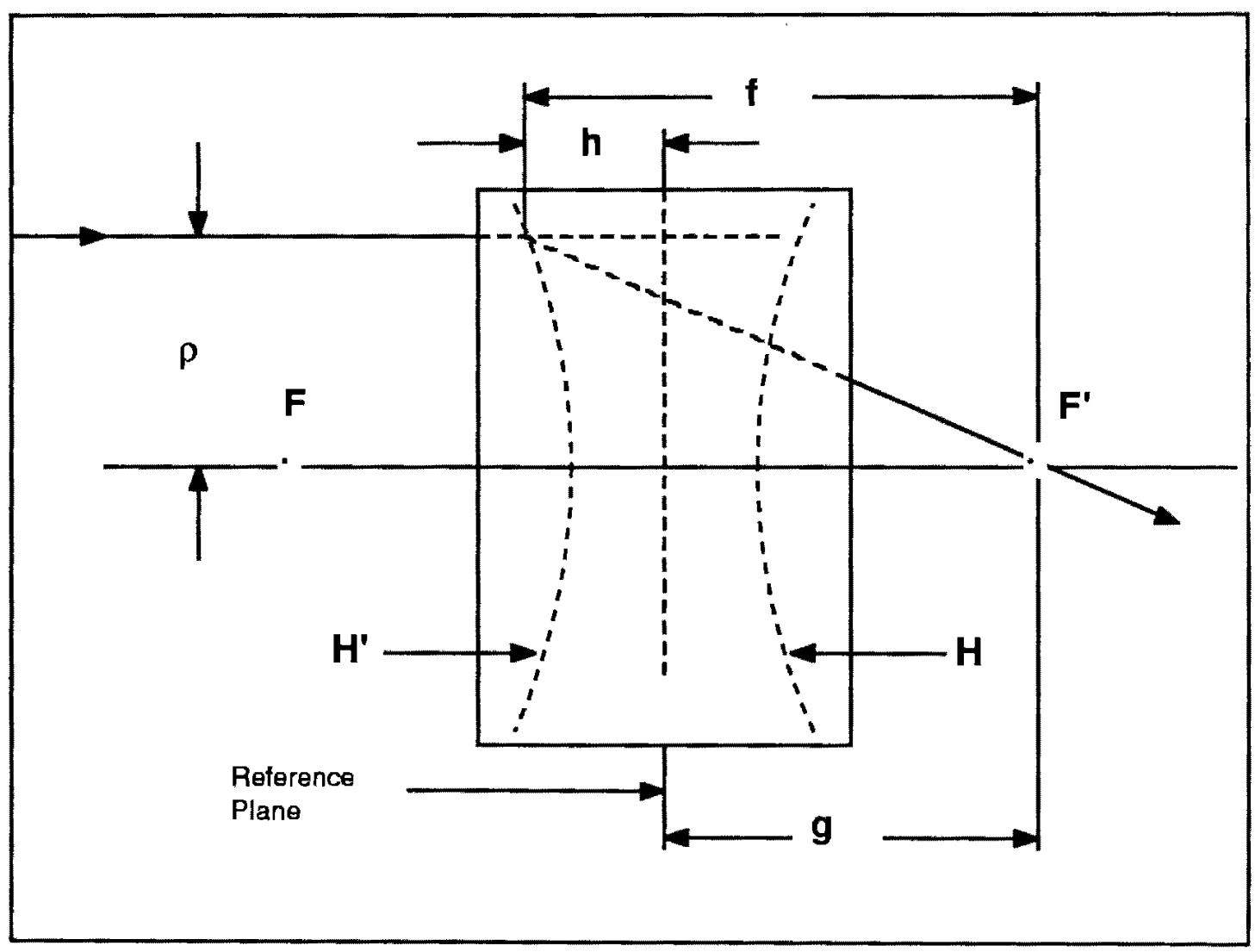

Figure 6. Ray trace for a distant source 
$g_{0}$, and the variations of focal length and focal distance can be described to second order by

$$
\begin{aligned}
& \frac{\Delta f}{f_{0}}=S_{f} \frac{\rho^{2}}{f_{0}^{2}}=S_{f}\left(1+\frac{1}{m_{0}}\right)^{2} \alpha^{2} \\
& \frac{\Delta g}{f_{0}}=S_{g} \frac{\rho^{2}}{f_{0}^{2}}=S_{g}\left(1+\frac{1}{m_{0}}\right)^{2} \alpha^{2}
\end{aligned}
$$

Where $\rho$ is the radial offset of the incoming ray from the axis at the principle surface, and $S_{f}$ and $S_{g}$ are the dimensionless spherical aberration constants. Expressing these coefficients in terms of the experimental quantities gives

$$
\begin{gathered}
S_{f}=-\frac{\left[\left(1+m_{0}^{2}\right) C_{1}-m_{0} C_{2}\right]}{\left(1-m_{0}^{2}\right)} /\left(\rho_{1} / f_{0}\right)^{2} \\
S_{g}=-\frac{\left(C_{2}-2 m_{0} C_{1}\right)}{\left(1-m_{0}^{2}\right)} /\left(\rho_{1} / f_{0}\right)^{2}
\end{gathered}
$$

where $C_{1}=\beta-\beta^{\prime} / M_{0}^{\prime}, C_{2}=c_{0} \beta^{\prime} / f_{0}$ and $\rho / f_{0}=\left(1+1 / m_{0}\right) \alpha_{1}$ where $\alpha_{1}=\varepsilon_{1} / a$.

\section{APPARATUS AND PROCEDURE}

An electron optical bench is used to hold the lens and other components in alignment with respect to each other. The bench is essentially a long horizontal vacuum chamber with veeways to support electron optical components, mechanical linkages to allow the components to be moved from outside the bench and electrical feedthroughs to provide the voltages needed by the source and lenses. An oblique end-view of the bench is shown in figure (7) with the components in place. At the far end in the veeways is a triode electron gun which served as the source for the experiment. Partly visible in the front is an aluminized phosphor-coated fiber optics window which allows the shadow images to be seen and recorded from ouside the bench. The disassembled three-ring lens is shown in figure (8). The three machined brass electrodes are arranged in order below the rexolite spacers that hold them in place and the lens housing is to the left. A scale drawing of the lens assembly is 


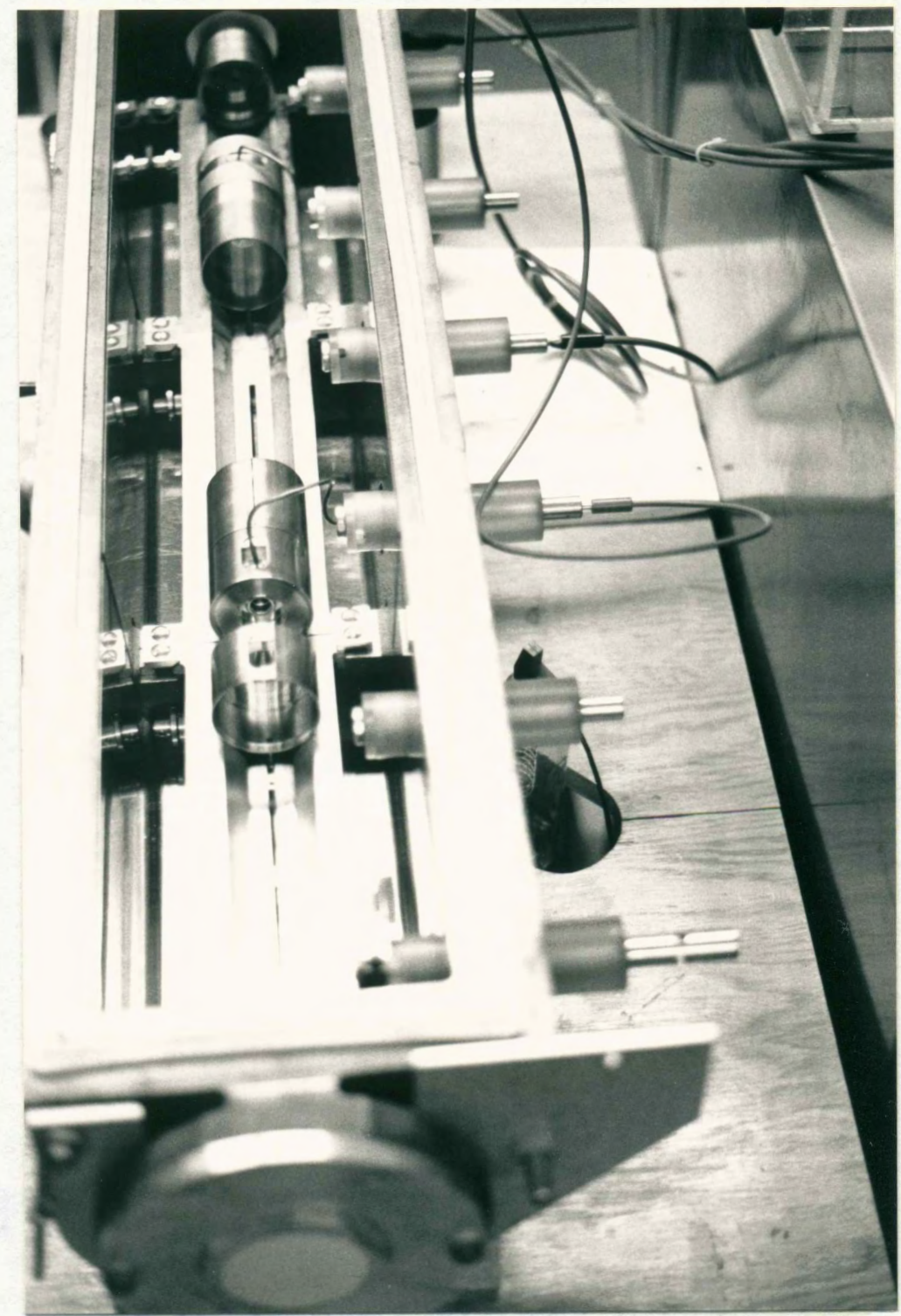

Figure 7. Electron optical bench with components 


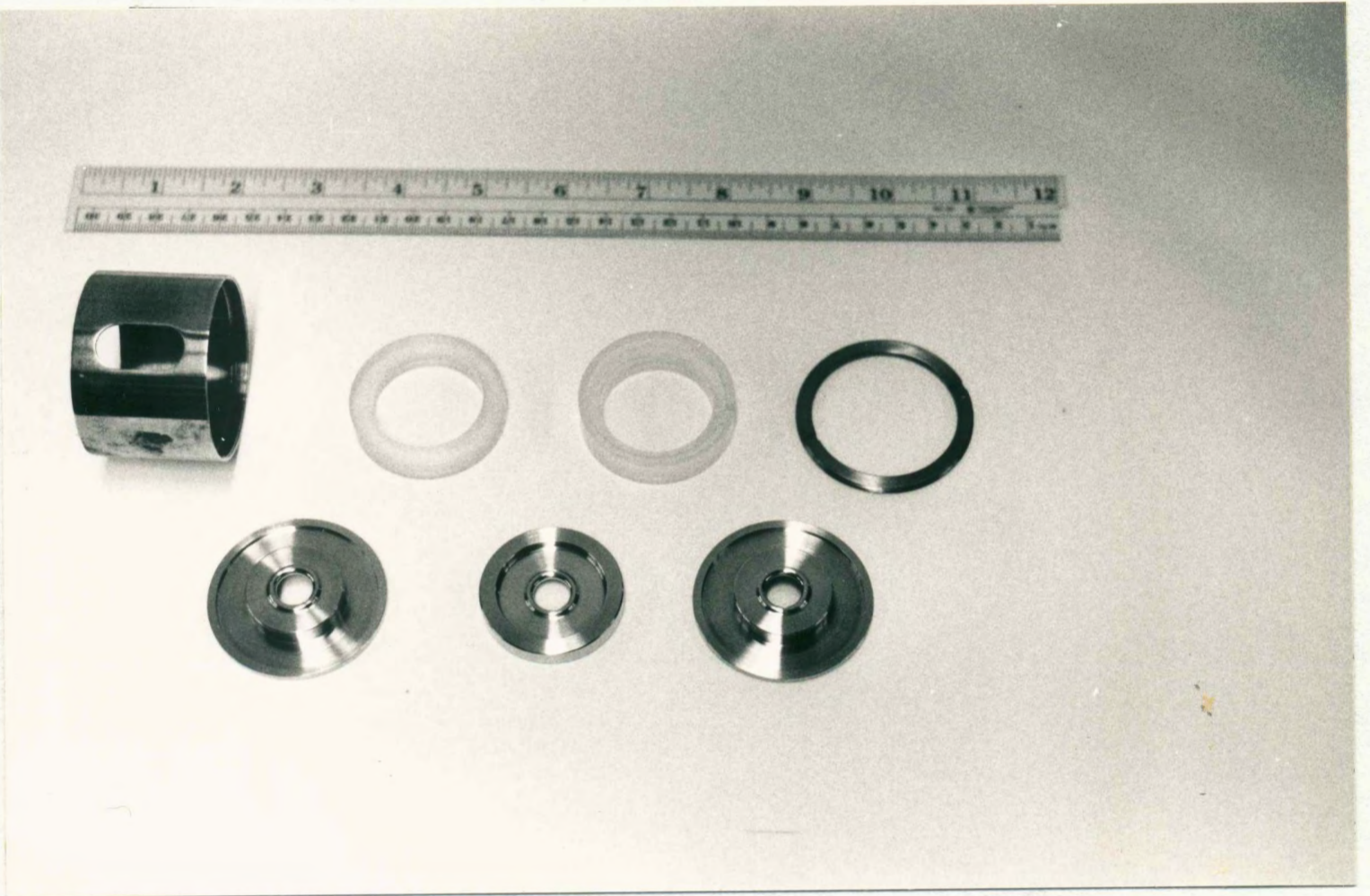

Figure 8. Three-ring lens disassembled 
given in figure (9) for completeness. The electron beam begins at the triode gun and then passes through a condenser lens which focuses it onto an aperture. This aperture serves as the effective source for the experiment. The beam then passes through the front grating, three-ring lens and rear grating before it finally reaches the fiber-optics window where a visible image is formed.

The experimental focal properties of the three-ring lens were determined for six different lens voltages. The accelerating potential was $20 \mathrm{kV}$ for all cases. The ratios of lens potential to accelerating potential, $V_{l} / V_{c}$, used were $1.0, .95, .90, .85, .80$ and .75. The bench pressure was less than $10^{-4}$ torr. For the first lens voltage, $V_{l} / V_{c}=$ 1.0 , the front grating position was adjusted so that about 20 grating shadows were observed and was not changed for the rest of the experiment. For each voltage the position of the rear grating was adjusted so that: 1) it was close enough to the image formed by the lens so that distortion was measurable and 2) enough shadow images were visible to reduce the error due to individual variations in the grating spacings. To accomplish this it was necessary in some cases $\left(V_{l} / V_{c}=.85, .80, .75\right)$ to place the grating on the lens side of the source image. This caused the distortion in the rear grating shadow to change from barrel to pincushion. Photographic film was placed in contact with the outside of the fiber optics window to record the pattern for each run. The recorded patterns were then measured and the data analyzed to find the focal properties for each case.

\section{SAMPLE CALCULATION AND RESULTS}

The data and calculations are included here for the case where $V_{l} / V_{c}=.95$ to demonstrate how the experimental values $f_{0}, g_{0}, S_{f}$ and $S_{g}$ were obtained. The shadow pattern for this case is shown in figure (10) and the data taken from the pattern is listed in table (II). The positions of the shadows cast by the front grating 


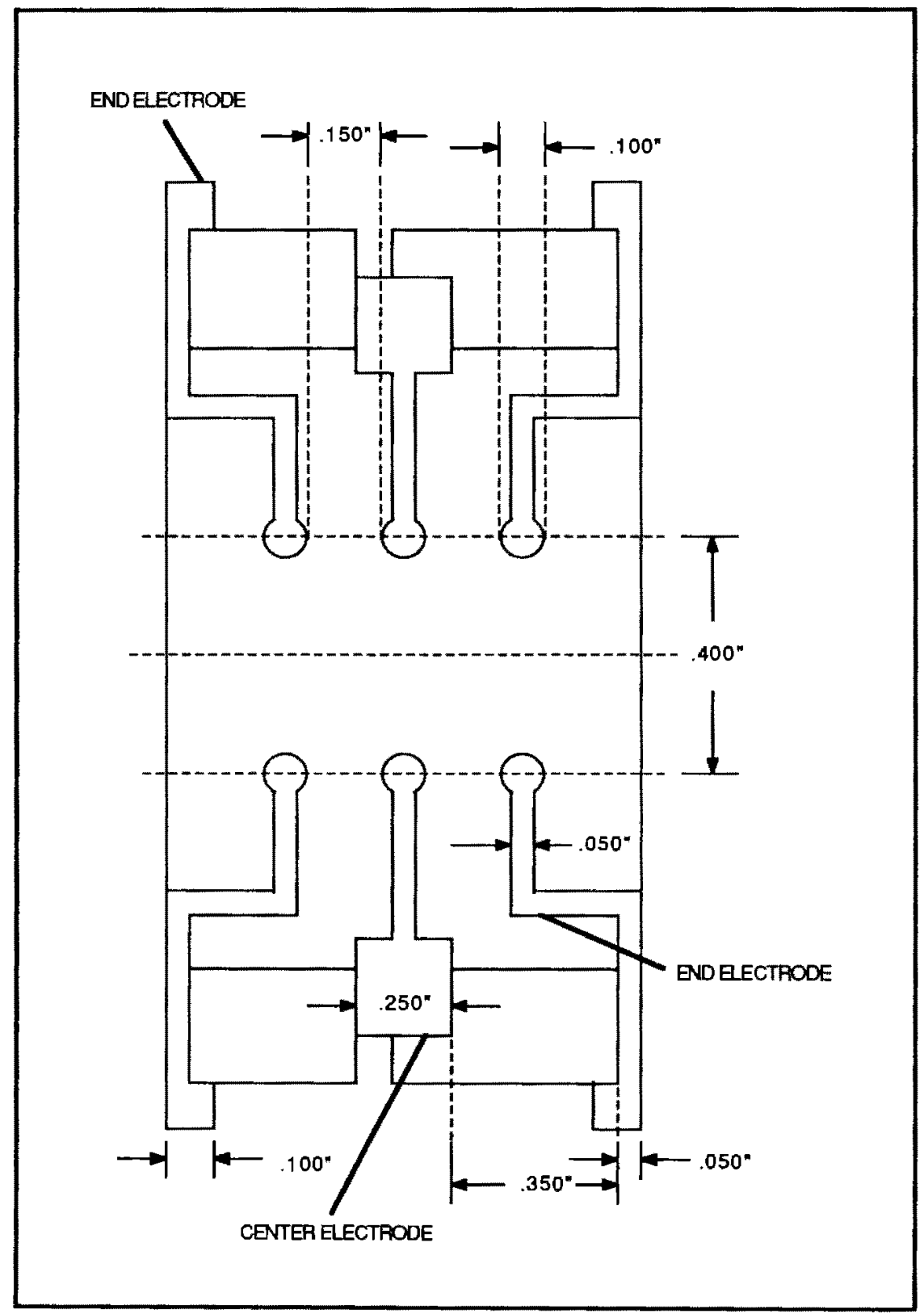

Figure 9. Scale drawing of lens assembly. 


$$
\begin{aligned}
& \text { \#2 3-renglax } \\
& V_{2} / V_{c}=0.95
\end{aligned}
$$

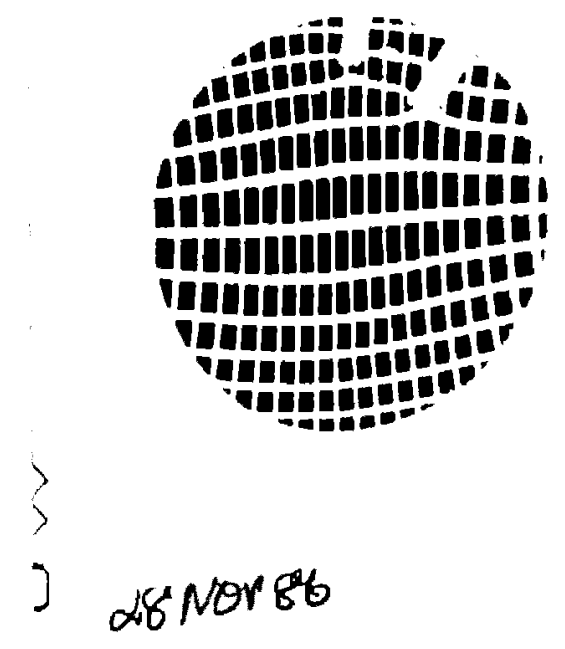

Eigure 10 Grating shadow pattern. Distortions in the pattern (otherwise a grid of rectangles) are manifestations of the lens aberrations. The two protrusions on top are from clips which held the gratings in place. 


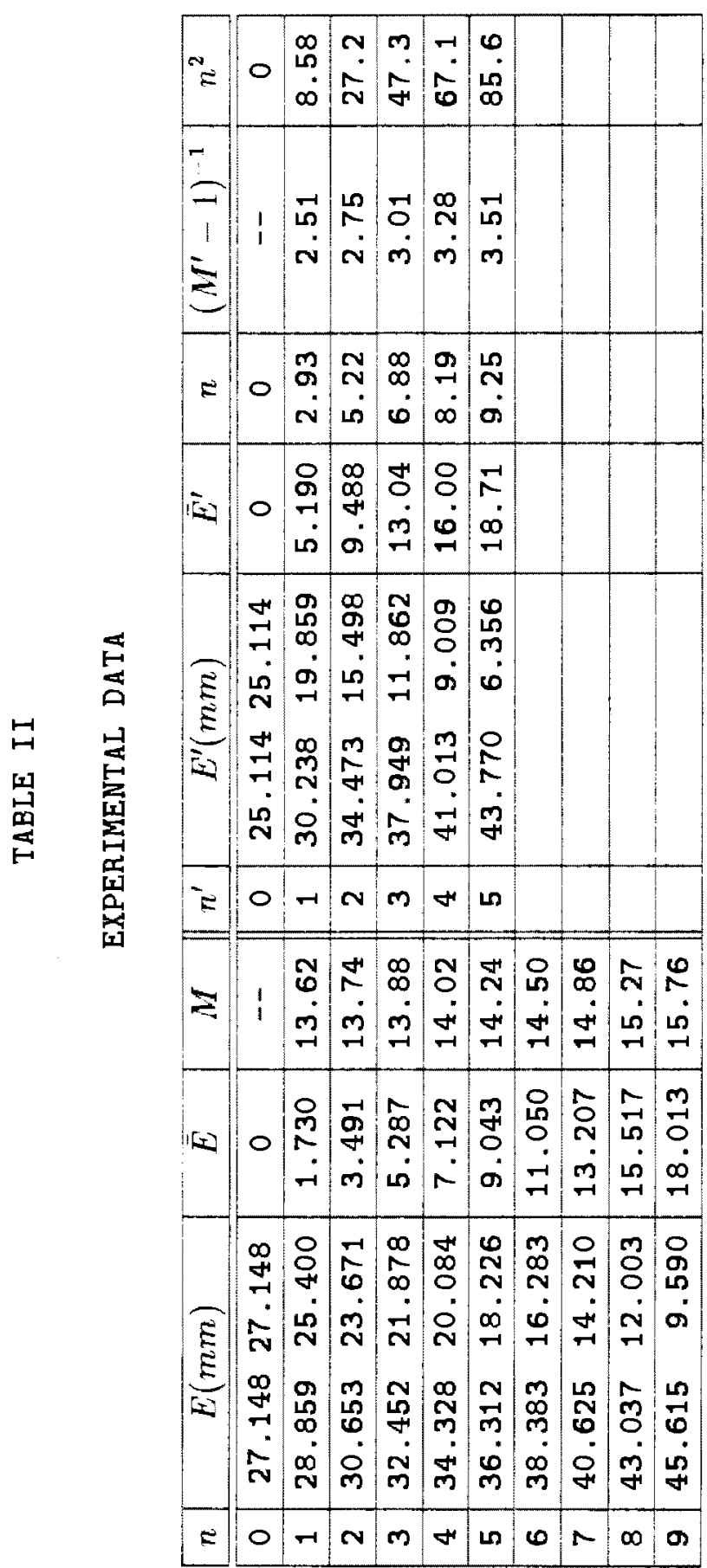


are known as a function of the front grating index $n$ and the positions on either side of the center are averaged to give $\bar{E}$. Likewise the positions of the shadows cast by the rear grating $E^{\prime}$ are known as a function of the rear grating index $n^{\prime}$ and are averaged to give $\bar{E}^{\prime}$. The relationship between $n$ and $n^{\prime}$ is found by plotting $\bar{E}$ vs $n$ and $\bar{E}$ vs $n^{\prime}$ as shown in figure (11). Now the shadow magnifications are found from $M=\bar{E} / n e$ and $M=E / n^{\prime} e$, where the spacing on the electron bar grids used is $e=125 \mu m$, and plots are made of $M$ vs $n^{2}$ and $\left(M^{\prime}-1\right)^{-1}$ vs $n^{2}$. These plots are shown in figures (12) and (13) and are linear as expected. The slope and intercept values from the plots are used with equations $(60)$ and (61) to give the paraxial magnifications and distortion coefficients which are

$$
\begin{gathered}
M_{0}=13.6 \\
M_{0}^{\prime}=43.0 \\
\beta=1.92 \times 10^{-3} \\
\beta^{\prime}=5.63 \times 10^{-3}
\end{gathered}
$$

The measured distances corresponding to figure (5) for this case are

$$
\begin{aligned}
& a=11.370 \mathrm{in} \\
& z=12.355 \mathrm{in} \\
& b=1.846 \mathrm{in} \\
& d=17.196 \mathrm{in}
\end{aligned}
$$

and finally these values are used in equations (55) through (64) to obtain

$$
f_{0}=1.259
$$




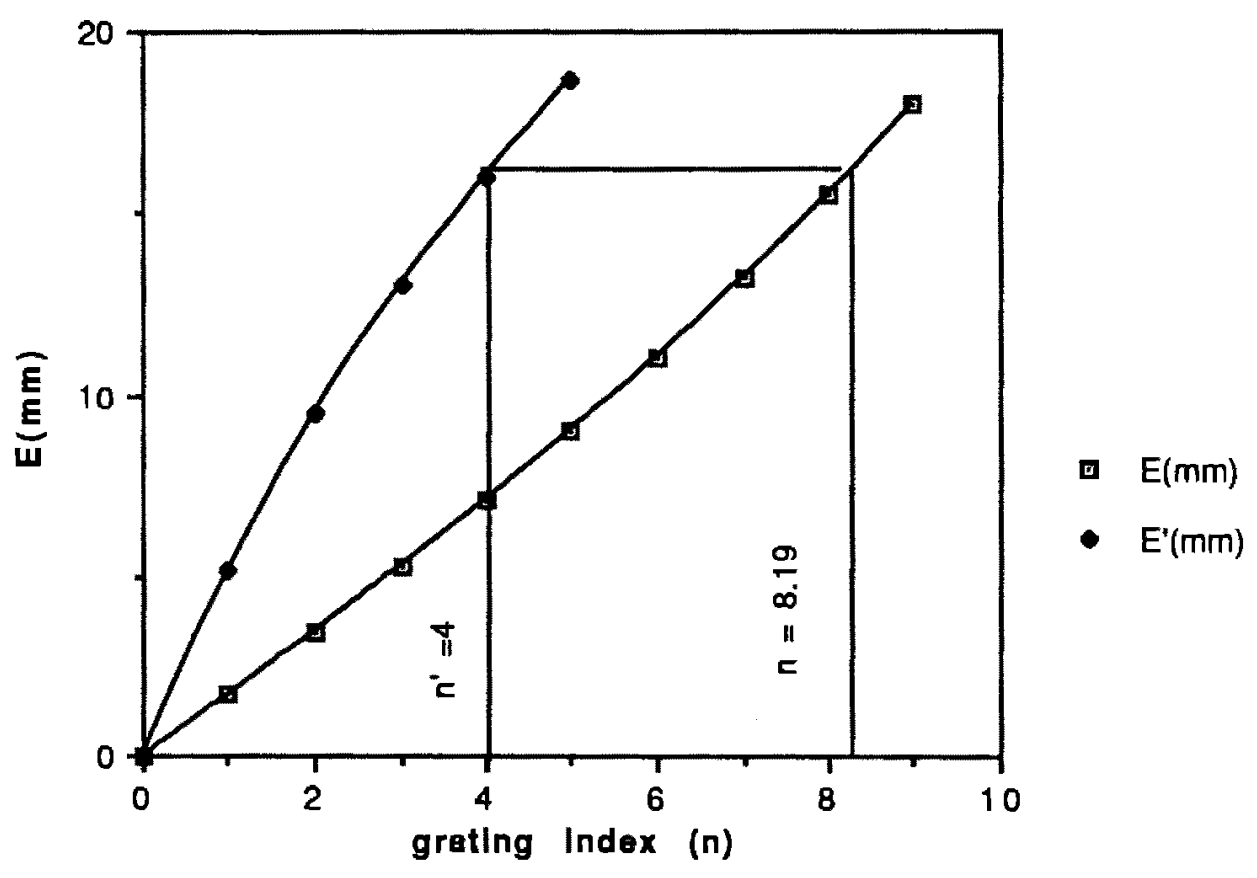

Figure 11. Method of converting $n$ ' to $n$ 


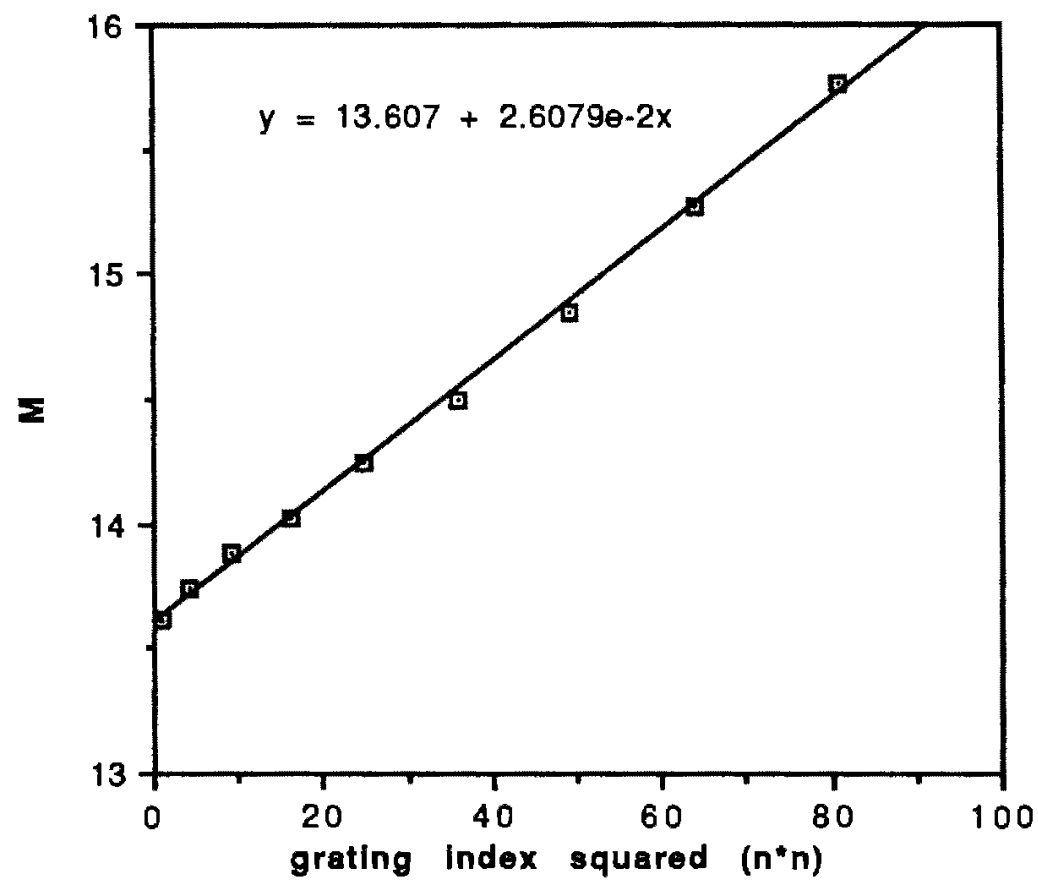

Figure 12. Magnification plot for front grating 


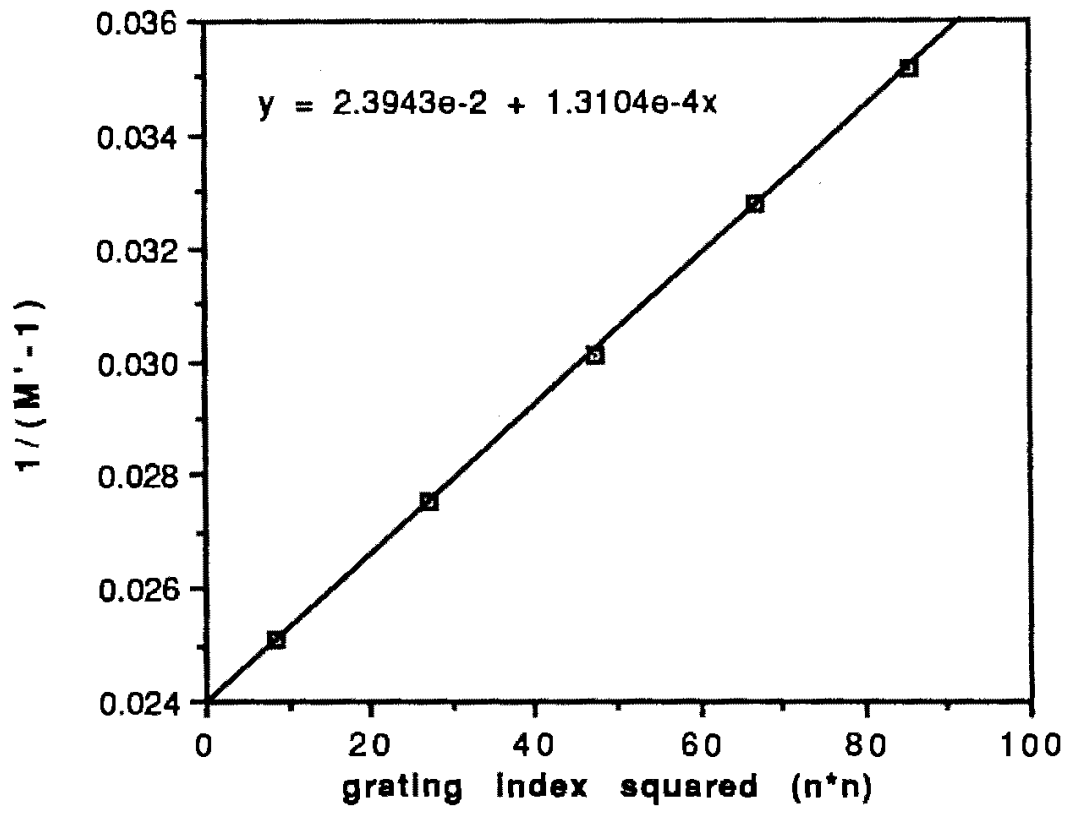

Figure 13. Magnification plot for rear grating. 


$$
\begin{gathered}
g_{0}=1.293 \\
S_{f}=-87.6 \\
S_{g}=-77.9
\end{gathered}
$$

Most of the error in this method is due to variations in the grating spacings. These errors are carried through into the graphs used for finding the shadow magnifications and the distortion coefficients. It is for this reason that the effort was made to include as many grating shadows as possible. If the variations are randomly distributed then the intercept errors should scale down with $1 / \sqrt{N}$ where $N$ is the total number of grating shadows measured. Another way to reduce this error is to measure the individual grating spacings and use the individual $e$ values. Table (III) gives the experimental focal properties for six voltage ratios from $V_{l} / V_{\mathrm{e}}=.75$ to $V_{l} / V_{\mathrm{e}}=1.00$. 
TABLE III

EXPERIMENTAL FOCAL PARAMETERS

\begin{tabular}{|c||c|c|c|c|}
\hline$V_{l} / V_{c}$ & $f_{0}(i n)$ & $g_{0}(i n)$ & $S_{f}$ & $S_{g}$ \\
\hline \hline 1.00 & 1.06 & 1.07 & -64.8 & -50.3 \\
\hline 0.95 & 1.26 & 1.29 & -87.6 & -77.9 \\
\hline 0.90 & 1.50 & 1.50 & -121 & -65.3 \\
\hline 0.85 & 1.78 & 2.85 & -158 & -167 \\
\hline 0.80 & 1.99 & 2.08 & -177 & -228 \\
\hline 0.75 & 2.39 & 2.56 & -179 & -433 \\
\hline
\end{tabular}




\section{CONCLUSIONS}

Graphs of the predicted and experimentally measured values for focal length, focal distance, and the spherical aberrations of these quantities are shown in figures (14),(15),(16) and (17) in pairs for direct comparison. The two sets of data show reasonable agreement. Some offset is expected because the theoretical study neglected two effects which are known to be present in the experiment : 1) polarization of charge on the electrodes and 2) the presence of the disc-like extentions on the electrodes. The main effect of charge polarization is to shift the centroids of the charges on the rings so that they are closer together. In the theoretical study this is equivalent to placing the hoops closer together which tends to increase the focal length and focal distance, and therefore is clearly not the dominating effect. The disc-shaped extensions on the electrodes, on the other hand, give the lens a larger total capacitance which increases the charge present on each electrode. This could also increase the charge residing on each ring, thereby increasing the strength of the lens. However, without a more detailed calculation it is difficult to say just how the increased charge distributes itself, i.e. whether it is mostly on the ring or the disc portion. The experimental results suggest that it is on the ring.

The manner in which the focal properties change with voltage is consistent between the two sets of curves. Figure (17) shows that the focal distance aberration is undercorrected, which is true for all electrostatic lenses. From the theoretical data it is evident that the focal length $f$ is always greater than the focal distance $g$ and ,therefore, the principle surfaces $H$ and $H^{\prime}$ are crossed for all the voltages used. The experimental values, however, are not as clear on this point because the experimental error is greater than the actual difference between $f$ and $g$. The 


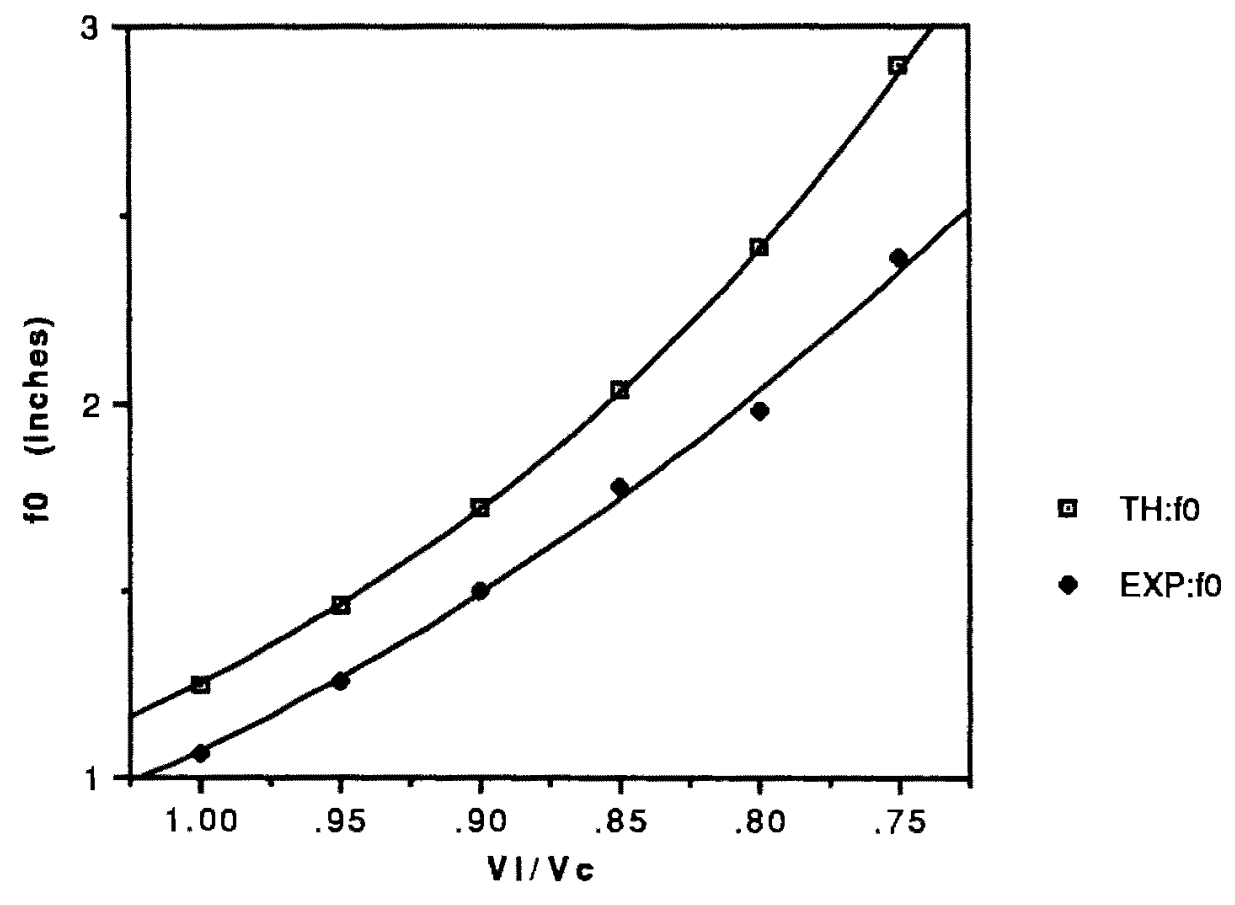

Eigure 14. Theoretical and experimental focal length 


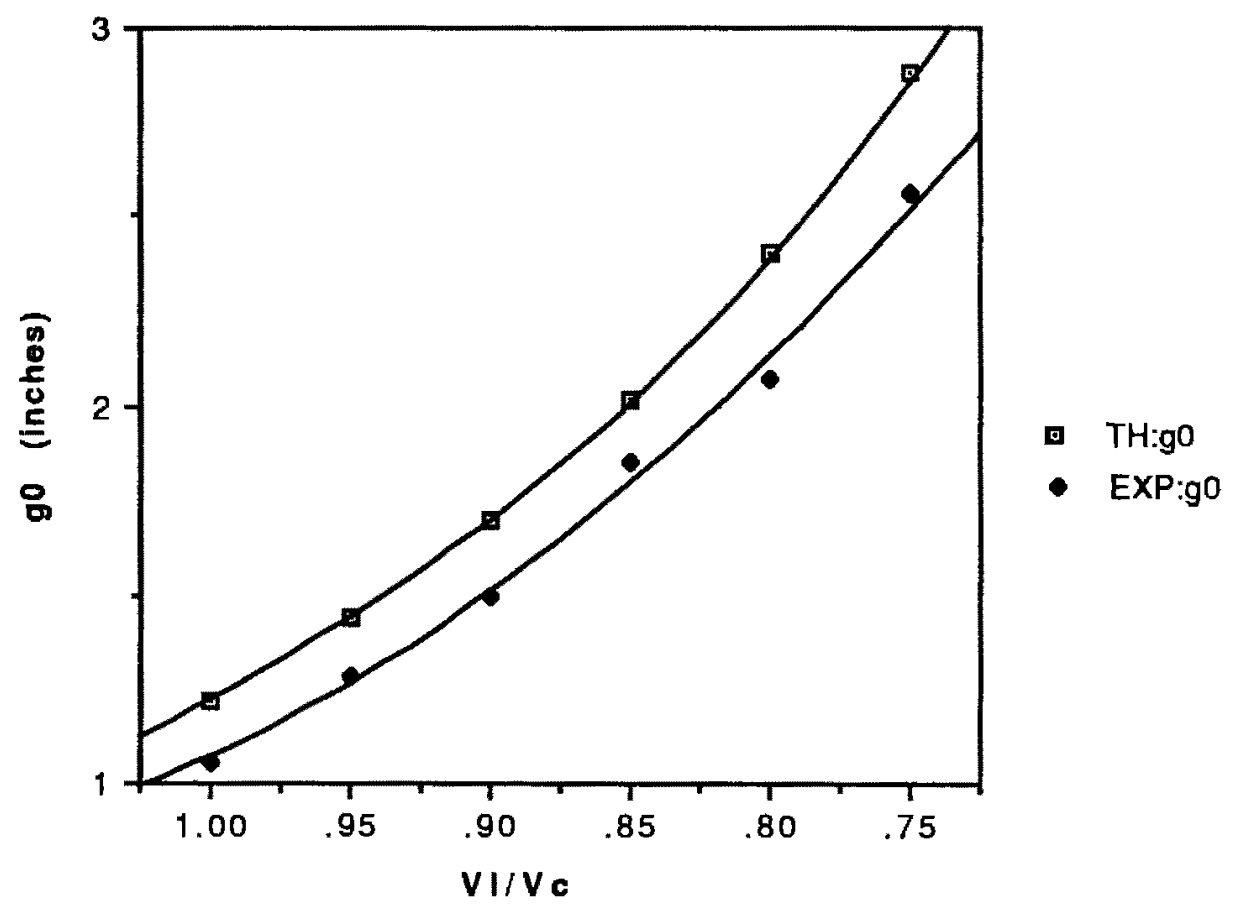

Eigure 15. Theoretical and experimental focal distance 


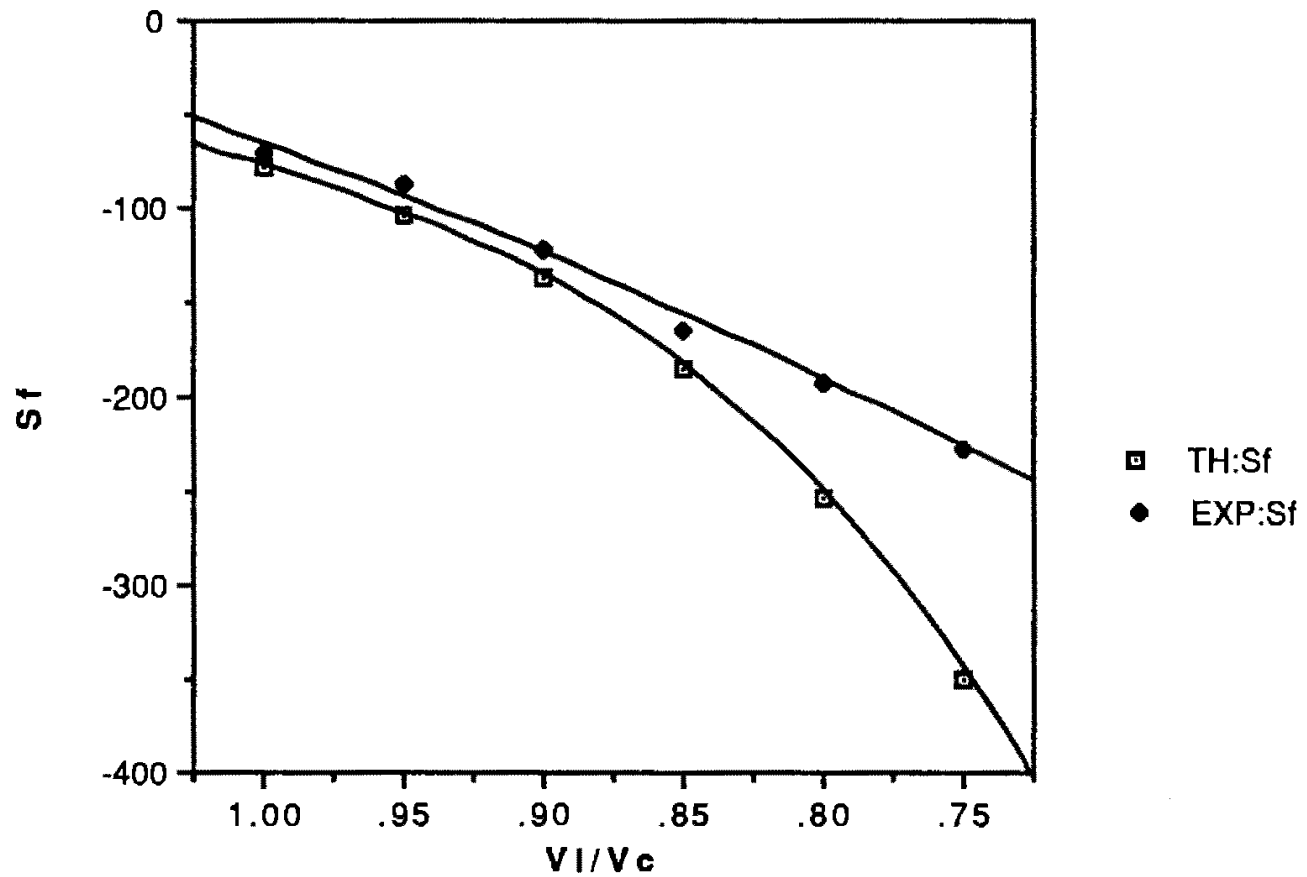

Eigure 16. Theoretical and experimental focal length aberration 


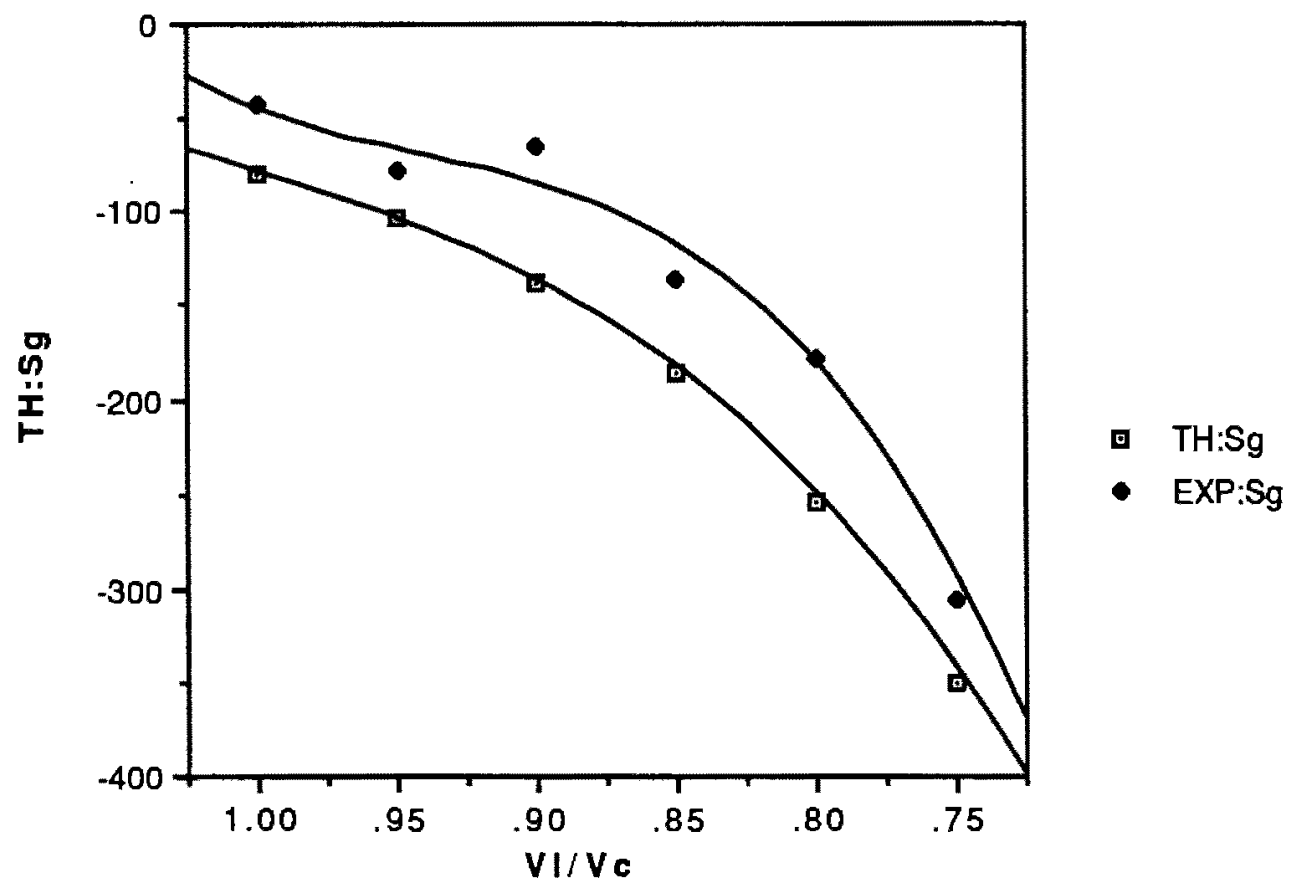

Figure 17. Theoretical and experimental focal distance aberration 
simplified charge density method used for the theoretical study shows good qualitative agreement with the experiment and with the known properties of electrostatic lenses. 


\section{GLASS LENS STUDY}

In this section the two-grating method discussed previously is used to measure the focal properties of a planoconvex glass lens, first with the flat side facing the source and then with the curved side facing the source. Throughout this section these two situations are referred to as configuration 1 and configuration 2 , respectively. The results from this experiment are compared with ray trace calculations based on the index of refraction, thickness and radius of curvature of the lens to provide the reader with a sense of the accuracy and reliability of the method.

\section{EXPERIMENT}

The two gratings used were photograph negatives with evenly spaced alternating clear and dark bars. These bars were measured with a traveling microscope and were found to have a spacing of $e_{1}=e_{1}^{\prime}=.1953 \mathrm{~cm}$. The grids were then mounted on glass plates whose thicknesses were $.30 \mathrm{~cm}$ each. The gratings were then mounted in holders so that they were oriented 90 degrees apart from each other. The plates, source, guide tube and lens were then mounted on an optical bench with a grating on either side of the lens. The source used was a concentrated arc lamp which produced a very bright (bluish) small circular spot at the end of its $3.58 \mathrm{~cm}$ bulb. The screen was in contact with the edge of the optical bench which was $3.8 \mathrm{~cm}$ from the $125 \mathrm{~cm}$ mark on the bench. Photographs of the components and the experimental arrangement are shown in figures (18) and (19). The distances between elements were all measured to within $0.5 \mathrm{~mm}$. The description up to this point refers to configuration 1 . For configuration 2 all the elements were in the same positions except that the lens was turned around so that its curved side faced 


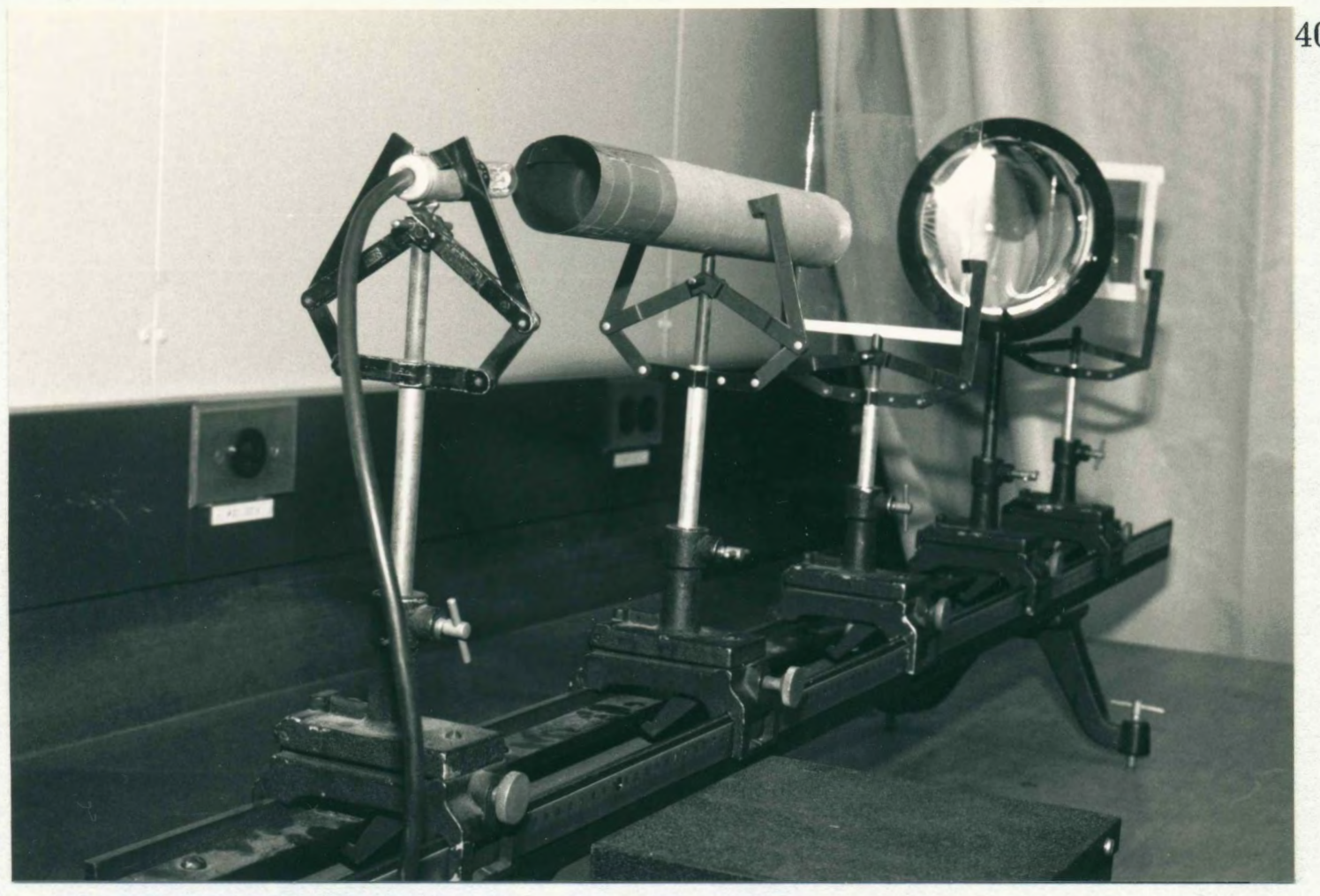

Figure 18. Experimental apparatus for glass lens study

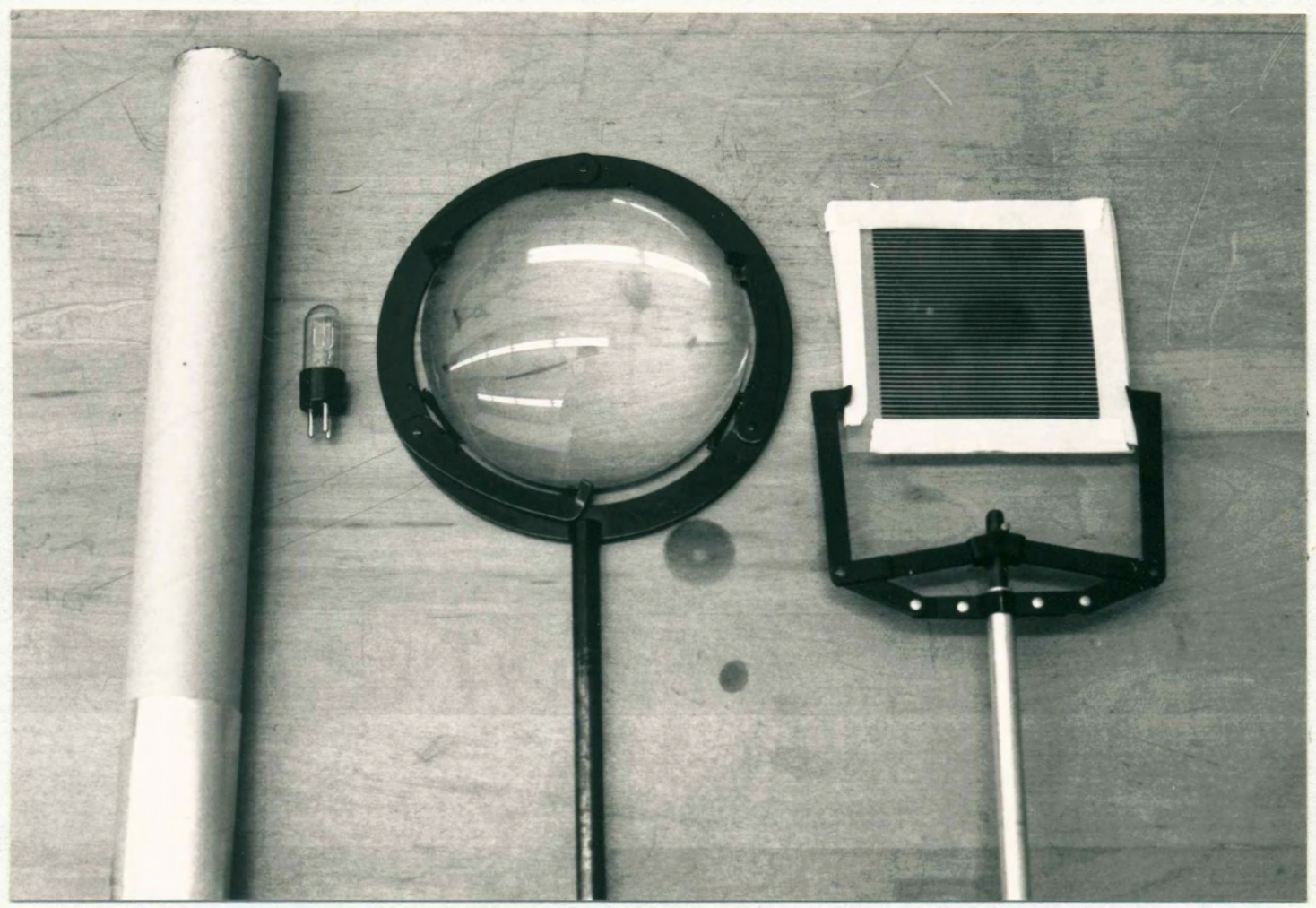

Figure 19. Experimental components for glass lens study 
the source while its flat side remained in the same position as before. Photographic print paper was then placed on the screen with the apparatus in a dark room and the source was turned on for about six minutes for each case. The prints containing the grating patterns were then developed and the distances between the shadows were obtained with a ruler and recorded.

\section{RESULTS}

The experimental and calculated focal properties of the lens for both configurations are presented in table (IV). The values for the discrepancies are also included because in this study, unlike the electron lens study, the theoretical and experimental results are expected to agree. The focal lengths are smaller than the focal distances in all cases, which is normal for a glass lens. The longitudinal spherical aberration $\Delta z^{\prime} / \rho^{2}$ for configuration 1 is about twice that for configuration 2 and both values agree very well with the ray trace calculations. This is due to the fact that the object is further away from the lens than the image and, therefore, the angles of deviation are minimized, in a least squares sense, when the curved side of the lens faces the source. The focal lengths had a $1 \%$ discrepancy, the focal distances agreed to about $2 \%$ and the spherical aberrations agreed to within $8 \%$. Much of this error can be attributed to the fact that a white light source was used. There is also error associated with the finite size of the source which manifests itself as "smearing" of the shadow patterns. It is expected that if a laser were used in place of the source the agreement would be even better. 
TABLE IV

GLASS LEMS RESULTS

TWO-GRATING METHOD

\begin{tabular}{|c||c|c|c|}
\hline Configuration & $f_{0}(\mathrm{~cm})$ & $g_{0}(\mathrm{~cm})$ & $\Delta z^{\prime} / \rho^{2} \mathrm{~cm}^{-1}$ \\
\hline \hline 1 & $15.75 \pm .42$ & $16.48 \pm .47$ & -.3415 \\
\hline 2 & $15.77 \pm .56$ & $16.44 \pm .66$ & -.1763 \\
\hline
\end{tabular}

RAY-TRACE CALCULATIONS

\begin{tabular}{|c||c|c|c|}
\hline Configuration & $f_{0}(\mathrm{~cm})$ & $g_{0}(\mathrm{~cm})$ & $\Delta z^{\prime} / \rho^{2} \mathrm{~cm}^{-1}$ \\
\hline \hline 1 & $15.60 \pm .69$ & $16.10 \pm .69$ & -.358 \\
\hline 2 & $15.60 \pm .69$ & $16.10 \pm .69$ & -.163 \\
\hline
\end{tabular}

\section{DISCREPANCIES}

\begin{tabular}{|c||c|c|c|}
\hline Configuration & $f_{0}(\%)$ & $g_{0}(\%)$ & $\Delta z^{\prime} / \rho^{2}(\%)$ \\
\hline \hline 1 & 1.0 & 2.3 & 4.7 \\
\hline 2 & 1.1 & 2.1 & 7.8 \\
\hline
\end{tabular}




\section{REFEREMCES}

1. Paszkonski, B,,1968, Electron Optics, Elsevier Publishing Company Inc., Ner York, P. 2

2. Busch, H., 1926, Annalen der Physik, Vol 81, p 974

3. De Broglie, P. H., 1924, 47, P 446

4. Davisson, C. J., and L. H, Germer, 1927, Phys. Rev., Dec. 30,705, Bell Laboratories Record, Vol. 4, No. 2, Apr. 1927, Pp. 257-260

5. Renau, A., F. H. Read and J. N. H. Brunt, 1881, J. Phys. E:Sci. Instrum. , 15, Pp. 347-54

6. Cruise, D. R., 1963 J. Appl. Phys., 34, Pp. 3477-9

8. Singer, B., and M. Braun, 1970, IEEE Trans. Elec. Devices, ED-17, p 926

9. Spangenberg, K., and L. M. Field, March 1942, Proceeding of the IRE, 30 , PP. 138-144

10. Rempfer, G. F., 1985, J. Appl. Phys., 57, p 2385 